\title{
38. DATA REPORT: MAGNETIC PROPERTIES AND MAGNETIC OXIDE MINERALOGY OF UPPER CRUSTAL ROCKS FROM HOLES 504B AND 896A ${ }^{1}$
}

\author{
Laura B. Stokking, ${ }^{2}$ Elizabeth A. Heise, ${ }^{2}$ Simon Allerton, ${ }^{3}$ and Horst-Ulrich Worm ${ }^{4}$
}

\begin{abstract}
We present the results of studies of magnetic mineralogy and magnetic properties of Holes 504B and 896A upper crustal rocks recovered during Leg 148 . The ratio of saturation remanence to saturation magnetization $\left(J_{R S} / J_{S}\right)$ ranges from 0.012 to 0.154 (mean $=0.06$ ) in samples from Hole 504B, consistent with pseudo-single-domain behavior. Hole 896A samples display a variable ratio of $J_{R S} / J_{S}$ that ranges from 0.012 to 0.154 (mean $=0.076$ ), reflecting the differences in grain morphologies and magnetic properties between pillow and massive units.

Intensity of natural remanent magnetization (NRM) ranges from 0.01 to $0.24 \mathrm{~A} / \mathrm{m}$ (mean $=0.09 \mathrm{~A} / \mathrm{m}$ ) in Hole 504B samples, less than that measured in samples recovered during other legs because the drilling-induced remanence imparted by the bottom-hole assembly was substantially less during Leg 148 . Volume susceptibility averages $0.032 \pm 0.029 \mathrm{SI}$, similar to that from upper parts of the dike sequence. In all samples examined from Hole 504B, high-temperature oxidation of primary titanomagnetite has produced lamellae or pods of magnetite and ilmenite. Hydrothermal alteration has further altered the minerals in some samples to a mixture of magnetite, ilmenite, titanite, and a high-titanium mineral (either rutile or anatase). Electron microprobe analyses show that magnetite lamellae are enriched in the trivalent oxides $\mathrm{Cr}_{2} \mathrm{O}_{3}$ (mean $=0.076$ wt $\%$ ), $\mathrm{Al}_{2} \mathrm{O}_{3}$ (mean $=0.566 \mathrm{wt} \%$ ), and $\mathrm{V}_{2} \mathrm{O}_{3}$ (mean $\left.=0.757 \mathrm{wt} \%\right)$, whereas divalent oxides are concentrated in ilmenite lamellae $(\mathrm{mean} \mathrm{MnO}=$ $1.997 \mathrm{wt} \%$ and mean $\mathrm{MgO}=0.073 \mathrm{wt} \%)$.

In samples from Hole 896A, NRM intensity ranges from 0.14 to $22.59 \mathrm{~A} / \mathrm{m}$ (mean $=5.06 \pm 4.91 \mathrm{~A} / \mathrm{m}$ ). Volume susceptibility averages $0.0272 \pm 0.0254 \mathrm{SI}$. Low-temperature oxidation of primary titanomagnetite has produced titanomaghemite exhibiting shrinkage cracks. Ulvöspinel content averages 0.508 and ranges from 0.217 to 0.647 . Titanomaghemite appears slightly enriched in $\mathrm{Al}_{2} \mathrm{O}_{3}$ (mean $\left.=1.697 \mathrm{wt} \%\right), \mathrm{Cr}_{2} \mathrm{O}_{3}$ (mean $\left.=0.064 \mathrm{wt} \%\right), \mathrm{V}_{2} \mathrm{O}_{3}$ (mean $\left.=0.673 \mathrm{wt} \%\right), \mathrm{MnO}(1.257 \mathrm{wt} \%$ ), and $\mathrm{MgO}$ $(0.577 \mathrm{wt} \%)$.
\end{abstract}

\section{INTRODUCTION}

During Ocean Drilling Program (ODP) Leg 148, Hole 504B was deepened from 2000 to $2111.0 \mathrm{~m}$ below seafloor (mbsf), and Hole $896 \mathrm{~A}$ was cored from 195.1 to $469 \mathrm{mbsf}$ ( $290 \mathrm{~m}$ into basement). Hole 504B lies $201 \mathrm{~km}$ south of the Costa Rica Rift (Fig. 1), the easternmost arm of the Galapagos Spreading Center in 6.6-m.y.-old crust (Cande and Kent, 1992). Hole 504B, drilled during eight Deep Sea Drilling Project (DSDP) and ODP legs, provides our most important in situ reference section for the structure of shallow ocean crust. Drilling results of previous legs to Hole 504B are summarized in CRRUST (1982); Cann, Langseth, Honnorez, Von Herzen, White, et al. (1983); Anderson, Honnorez, et al. (1982); Anderson, Honnorez, Becker, et al. (1985); Leinen, Rea, et al. (1986); Becker, Sakai, et al. (1988, 1989), Becker et al. (1989); Alt et al. (1986); Becker, Foss, et al. (1992), and Dick, Erzinger, Stokking, et al. (1992). Site 896, 1 km to the south of Site 504, was drilled to examine local variability in volcanic stratigraphy and the effects of off-axis hydrothermal activity on basement alteration in an area of high heat flow.

We present the results of studies of magnetic mineralogy and magnetic properties of upper crustal rocks from Holes 504B and 896 A drilled during Leg 148. Allerton et al. (this volume) discuss the paleomagnetism of Holes 504B and 896A and Worm et al. (this volume) discuss the results of downhole magnetic logging, thermal

'Alt, J.C., Kinoshita, H., Stokking, L.B., and Michael, P.J. (Eds.), 1996. Proc. ODP, Sci. Results, 148: College Station, TX (Ocean Drilling Program).

${ }^{2}$ Ocean Drilling Program, Texas A\&M University Research Park, 1000 Discovery Drive, College Station, TX 77845-9547, U.S.A. stokking@ cook.tamu.edu

'Department of Geology and Geophysics, University of Edinburgh, The Grant Institute, West Mains Road, Edinburgh EH9 3JW, United Kingdom. simona@glg.ed.ac.uk

${ }^{4}$ Bundesanstalt für Geowissenschaften und Rohstoffe, Stilleweg 2, Postfach 510153, D-30631 Hannover, Federal Republic of Germany. properties of Leg 148 samples, and their implications for understanding the source of marine magnetic anomalies.

\section{BACKGROUND}

Understanding the structure, mineralogy, and chemical alteration processes in the oceanic crust are among the most important scientific goals of ODP and of Leg 148. Knowledge of the nature of the oceanic basement is derived mainly from studies of ophiolite complexes, remote geophysical surveys, and studies made on dredged or cored samples from the ocean floor. Only Hole 504B penetrates through the extrusive pillow lavas into the sheeted dike complex to provide a representative in situ section of the ocean crust.

The magnetic properties of rocks depend on many variables: the types of magnetic minerals present in the rock (which themselves reflect the initial rock composition and any alteration, thermal or chemical, that the rock has undergone), the concentrations and the grain volumes of the various magnetic minerals in the rock, and the magnetic domain state of those minerals. The predominant primary magnetic mineral in ocean crustal rocks is titanomagnetite, an inverse spinel that is a solid solution between iron- and titanium-bearing end members, which may also contain aluminum, chromium, magnesium, vanadium, manganese, and nickel. Secondary phases, such as titanomaghemite, ilmenite, magnetite, hematite, and titanite, are produced by thermal and chemical alteration of titanomagnetite. In some cases, secondary phases and alteration textures, that is, the exsolution of primary titanomagnetite into low-titanium titanomagnetite and high-titanium hemoilmenite (Haggerty, 1976; Smith et al., 1991), can be observed using reflected light microscopy (Ade-Hall et al., 1971; Johnson and Hall, 1978) or backscattered electron imaging in conjunction with electron microprobe analysis. In other cases, however, the exsolution lamellae are too fine to be observed optically but may 


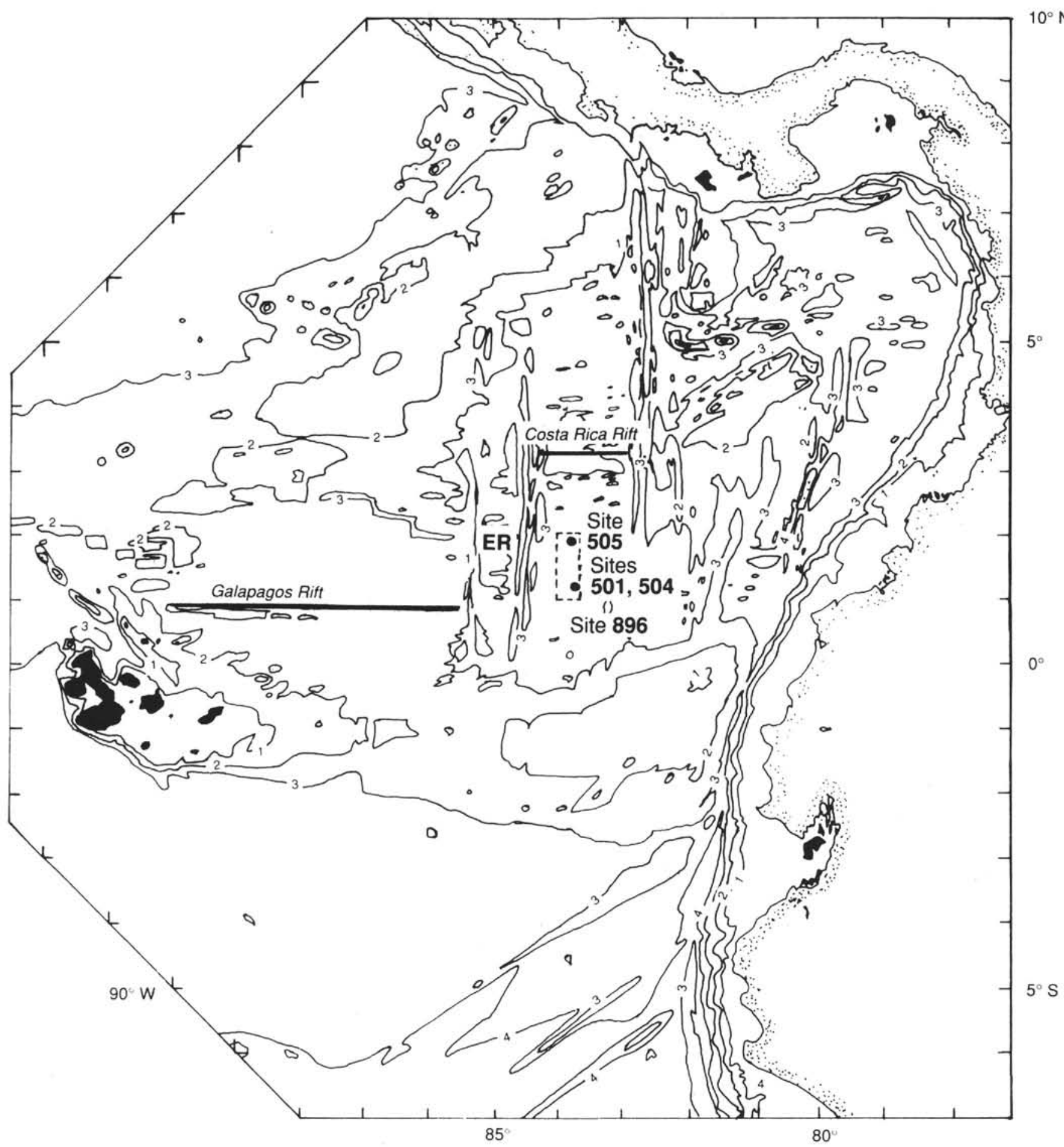

Figure 1. Location of Holes 504B and 896A (after Hobart et al., 1985).

be detected by the disagreement between the bulk composition of a grain (determined by electron microprobe analysis) and the Curie temperature (the temperature above which a mineral behaves paramagnetically and cannot retain a magnetic remanence) predicted on the basis of that bulk composition (Evans and Wayman, 1974; Rice et al., 1980; Smith and Banerjee, 1986; Smith et al., 1991).

Deuteric oxidation of titanomagnetites has been classified by Ade-Hall et al. (1968) and modified by Haggerty (1991). Hall and Fisher (1987) describe stages of hydrothermal alteration of titanomagnetites. According to Ade-Hall et al. (1968) and Haggerty
(1991), C1 grains are optically homogeneous titanomagnetite, C2 titanomagnetites contain some exsolved ilmenite lamellae along the (111) plane, C3 grains contain Ti-poor magnetite lamellae intergrown with exsolved ilmenite (up to $50 \%$ of grain), and $\mathrm{C} 4$ grains display mottling that represents the formation of ferrirutile or exsolved spinel. Hydrothermal alteration stages (Hall and Fisher, 1987) begin with $\mathrm{H1}$, in which there is no evidence of hydrothermal alteration. In $\mathrm{H} 2$ grains, fine titanite grains begin to replace ilmenite lamellae. Replacement of ilmenite lamellae by titanite is complete in $\mathrm{H} 3$ grains. Progression of alteration in $\mathrm{H} 4$ grains is marked by loss of 
Fe from magnetite lamellae. Anatase or rutile replaces $50 \%$ of $\mathrm{H} 5$ grains and $100 \%$ of $\mathrm{H} 6$ grains (Hall and Fisher, 1987).

In this study, magnetic minerals are identified and characterized on the basis of reflected and transmitted light microscopy in conjunction with electron microprobe analysis, thermal and alternating field demagnetization behavior, anhysteretic and isothermal remanence acquisition, and hysteresis parameters. Alternating field (AF) demagnetization yields the coercivity spectrum (a property that, assuming the sample contains a single type of magnetic carrier, is itself dependent on the grain-volume spectrum in the rock), and indicates whether the coercivity determined by measurement of hysteresis represents that of the carrier of remanence. Measurement of magnetic hysteresis (the magnetization behavior exhibited by a sample in the presence of an increasing magnetic field) will determine coercivity, saturation remanence, and saturation magnetization. These parameters, in conjunction with coercivity of remanence (a measurement of the reverse field required to remove saturation isothermal remanence), will then provide constraints on the domain state of the magnetic minerals in the sample (Dunlop, 1969).

\section{Site 504}

The Hole 504B basement section includes $571.5 \mathrm{~m}$ of pillow lavas and minor flows, underlain by a 209-m transition zone of mixed pillow lavas, thin flows and dikes, and $1056.0 \mathrm{~m}$ of sheeted dikes and massive units (Fig. 2; Alt, Kinoshita, Stokking, et al., 1993). Lithologic units identified during Leg 148 (Alt, Kinoshita, Stokking, et al., 1993 ) include sparsely to moderately phyric plagioclase-olivinepyroxene and olivine-plagioclase \pm pyroxene diabase. Average silicate grain size ranges from 0.4 to $1.0 \mathrm{~mm}$, whereas that of titanomagnetite ranges from 0.02 to $0.45 \mathrm{~mm}$. Exsolution lamellae of ilmenite are visible in the larger grains, and about half the units contain trace amounts of chrome spinel (Alt, Kinoshita, Stokking, et al., 1993).

Alteration is heterogeneous throughout the interval recovered during Leg 148 (Fig. 2; Alt, Kinoshita, Stokking, et al., 1993). Pervasive background alteration is slight to moderate, whereas more strongly altered regions form centimeter- to decimeter-sized patches and surround veins. Talc + magnetite, chlorite + quartz, actinolite \pm magnetite replace interstitial material, and titanite partly replaces igneous magnetite (Alt, Kinoshita, Stokking, et al., 1993).

Magnetic susceptibilities of shipboard samples average $0.018 \pm$ $0.013 \mathrm{SI}$, similar to values measured from dikes drilled during Legs 111,137 , and 140. The steeply inclined drilling-induced remanence that overprinted shallower dikes was not observed in Leg 148 cores: the bottom-hole assembly used on Leg 148 was new and had therefore never been magnafluxed (Alt, Kinoshita, Stokking, et al., 1993). Thus, natural remanent intensities measured on board ship (mean = $0.48 \pm 0.39 \mathrm{~A} / \mathrm{m}$ ) were lower, mean destructive fields were higher, and the properties measured were more representative of in situ values than those determined from dikes recovered during earlier visits to Hole 504B. A relatively weak viscous component was removed by alternating field demagnetization at $20 \mathrm{mT}$ (Alt, Kinoshita, Stokking, et al., 1993).

\section{Site 896}

Cores recovered from Hole $896 \mathrm{~A}$ contained massive basalt, pillow lavas, breccias, and dikes. The basalts are predominantly sparsely to highly phyric plagioclase-olivine basalts or olivine-plagioclase basalts, both commonly containing spinel (Fig. 3; Alt, Kinoshita, Stokking, et al., 1993).

The interval recovered from Hole $896 \mathrm{~A}$ is divided into two zones on the basis of igneous petrology and magnetic properties. The boundary between the two zones corresponds to the top of a thick sequence of massive units at $390 \mathrm{mbsf}$. In the upper section, plagioclase is the predominant phenocryst, whereas in the lower section, olivine dominates the phenocryst assemblage. Oxidative alteration, charac- terized by red alteration halos and by the replacement of olivine with smectite, is most visible in the massive units of the lower section (Alt, Kinoshita, Stokking, et al., 1993).

The intensity of natural remanence is relatively high in the upper part of the hole (shipboard samples: $11.9 \mathrm{~A} / \mathrm{m}$ ), the median destructive field is high (shipboard samples: $22.6 \mathrm{mT}$ ), and the bulk susceptibility is relatively low (shipboard samples: 0.015 SI). Stable inclinations measured from samples from the upper part are consistent and average $-9.5^{\circ} \pm 10.4^{\circ}$ (Allerton et al., this volume; Alt, Kinoshita, Stokking, et al., 1993).

NRM intensity is lower in the lower part of the hole (shipboard samples: $3.9 \mathrm{~A} / \mathrm{m}$ ), median destructive fields are lower (shipboard samples: $12.3 \mathrm{mT}$ ), and bulk susceptibilities are higher (shipboard samples: $0.033 \mathrm{SI}$ ). Alternating field demagnetization generally isolates two components: a low-coercivity phase, probably carried by titanomagnetite and/or titanomaghemite; and a higher coercivity phase, probably carried by secondary iron oxides and oxyhydroxides (Alt, Kinoshita, Stokking, et al., 1993). Stable inclination data vary widely and are discussed in Allerton et al. (this volume).

\section{METHODS}

Hysteresis behavior of 14 samples was measured using a Princeton Applied Research vibrating sample magnetometer in the Paleomagnetic Laboratory in the Geology Department of the University of California, Davis. Sample sizes in this study ranged from 0.005 to $0.0098 \mathrm{mg}$. Hysteresis measurements are made by applying an increasing magnetic field to a sample, then reversing the applied field, while continually measuring the magnetization of the sample as it responds to the changing applied field. A plot of applied field vs. sample magnetization results in a loop, the shape of which is determined by the chemical composition, microstructure, and particle orientation of the magnetic material within the sample (Stacey and Banerjee, 1974; Day et al., 1977; Cisowski, 1980). The hysteresis parameters saturation remanence $\left(J_{R S}\right)$, saturation magnetization $\left(J_{S}\right)$, and coercivity $\left(H_{C}\right)$ are obtained from the hysteresis loop. Coercivity of remanence $\left(H_{C R}\right)$ was determined using the vibrating sample magnetometer on the samples whose hysteresis behavior was studied. A field of $1.2 \mathrm{~T}$ was used to saturate the samples.

Before proceeding with alternating-field (AF) demagnetization, intensities of natural remanent magnetization (NRM) of 19 samples from Hole 504B and 71 samples from Hole 896A were measured on board the JOIDES Resolution (Alt, Kinoshita, Stokking, et al., 1993). NRM intensities of 15 additional samples from Hole 504B and of 41 additional samples from Hole 896A were measured in the Paleomagnetic Laboratory at Texas A\&M University (TAMU). On the Resolution, both a Molspin spinner magnetometer and a 2-G Enterprises (Model 760R) pass-through cryogenic rock magnetometer were used. At TAMU, NRM intensities were measured on a three-axis CTF cryogenic magnetometer housed in a shielded room at TAMU, and corrected by sample volume. Magnetic susceptibilities of discrete samples on board ship were determined using a Kappabridge KLY-2. Magnetic susceptibilities of samples in this study were measured at TAMU using a Bartington Instruments Susceptibility Meter at a frequency of $0.47 \mathrm{kHz}$, and corrected by sample volume. Three samples from Hole $896 \mathrm{~A}$ were thermally demagnetized using a Schonstedt thermal demagnetizer (Model TSD-1) at TAMU. Samples were heated at temperature intervals between $20^{\circ} \mathrm{C}$ and $50^{\circ} \mathrm{C}$ up to $600^{\circ} \mathrm{C}$ and the remanence measured between steps.

Studies of acquisition of isothermal remanent magnetization (IRM) and anhysteretic remanent magnetization (ARM) were performed on board ship during Leg 148 (Alt, Kinoshita, Stokking, et al., 1993). Shipboard ARMs were produced using alternating fields up to $100 \mathrm{mT}$ and a DC-biased field of $0.032 \mathrm{mT}$ (Alt, Kinoshita, Stokking, et al., 1993). Few samples, however, were fully saturated by the maximum applied field. 


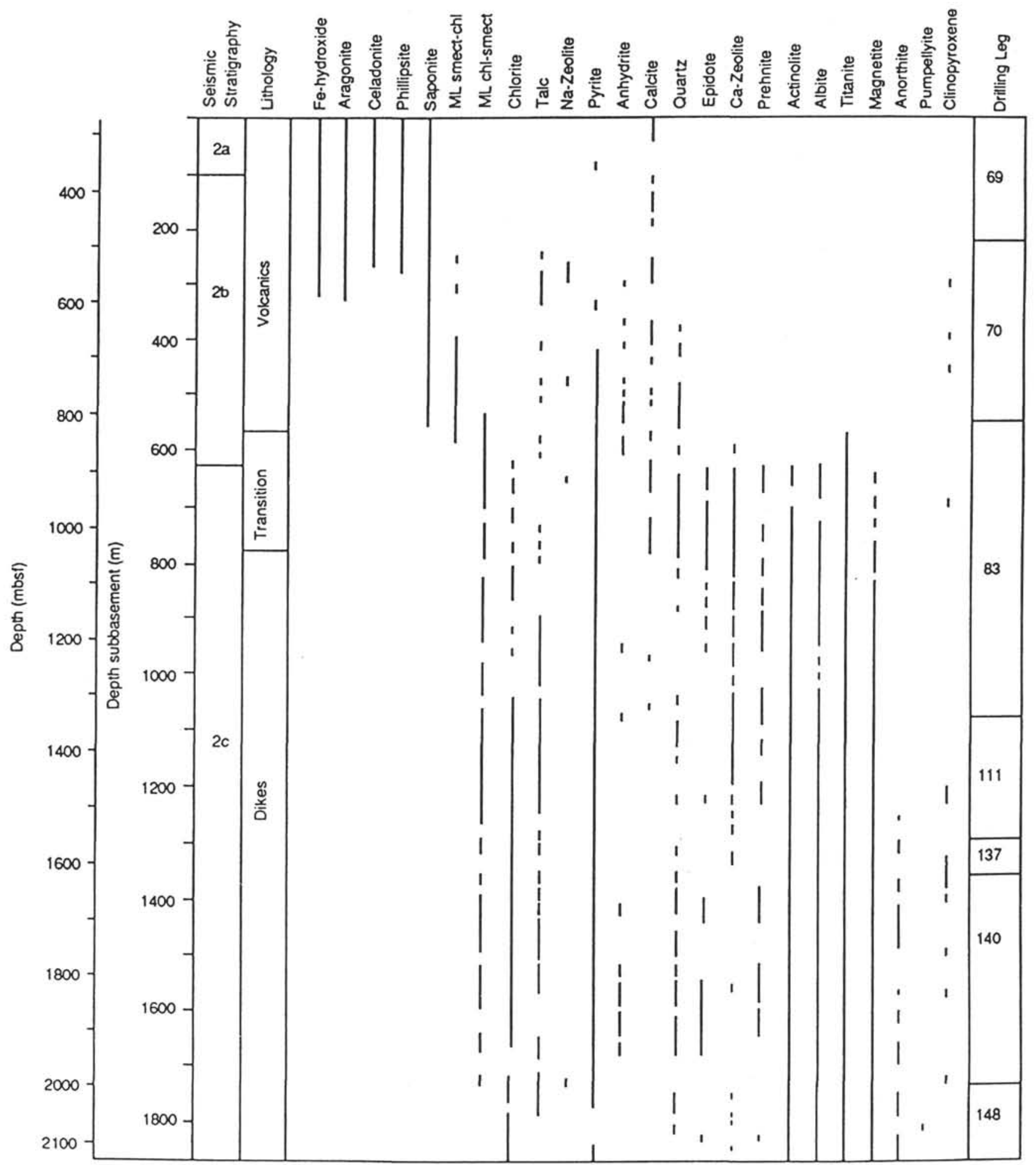

Figure 2. Distribution of secondary mineralogy, seismic stratigraphy, and lithology in Hole 504B (from Alt et al., 1986; Becker, Sakai, et al., 1989; Alt, Kinoshita, Stokking, et al., 1993).

On board ship, an AF demagnetizer (Model 2G600) capable of producing an alternating field up to $20 \mathrm{mT}$ was used on-line with the pass-through cryogenic magnetometer. AF demagnetization at higher fields was performed using a single-axis Schonstedt Geophysical Specimen Demagnetizer (Model GSD-1) capable of producing alternating fields up to $100 \mathrm{mT}$. At TAMU, samples were AF demagne- tized using Schonstedt Geophysical Specimen Demagnetizer (Model GSD-1). Samples were analyzed using the cryogenic magnetometers between AF steps.

After AF demagnetization at TAMU, a maximum ARM was subsequently imparted to 52 samples using an alternating field of 100 $\mathrm{mT}$ and $\mathrm{a} \mathrm{DC}$ bias field of $0.05 \mathrm{mT}$. The Schonstedt AF demagnetizer 


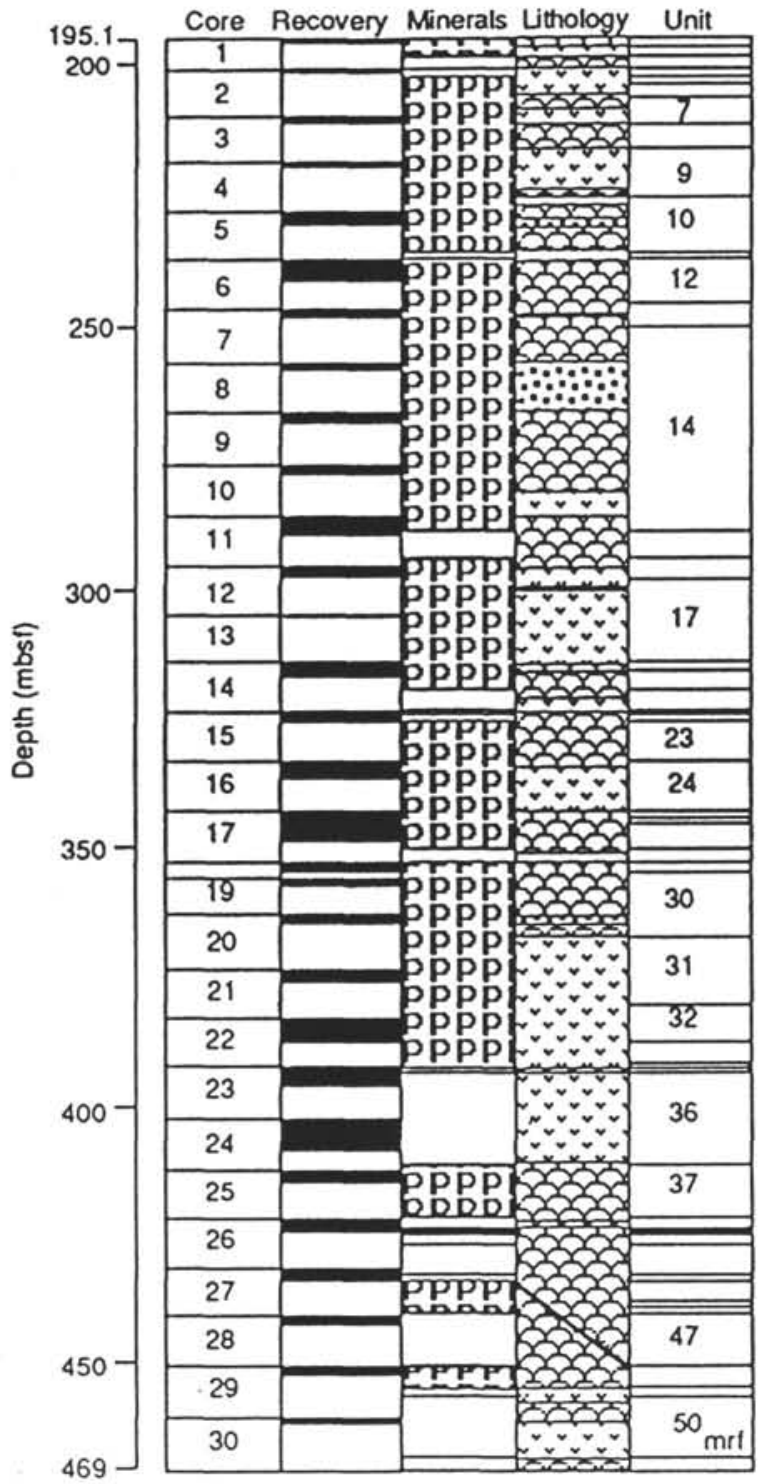

Figure 3. Drilling and recovery at Hole 896A. Black bars show recovery relative to core length. The dominant phenocryst is olivine, except where the symbol " $\mathrm{P}$ " in the minerals column is employed to indicate that plagioclase is dominant. Symbols in the lithology column indicate eruptive type: pillow basalts = convex upward, massive = "V", and breccia = solid. A mixture of pillow basalts, pillow-breccias, and breccias is indicated by a solid diagonal line (Alt, Kinoshita, Stokking, et al., 1993).

and an anhysteretic magnetizer constructed at TAMU by W. Sager were used to produce the ARM. The ARM of 16 samples was then AF demagnetized so the demagnetization behavior could be compared with that of NRM and IRM.

An IRM was applied at a maximum field of $1.0 \mathrm{~T}$ to 53 samples in the study using an ASC, Inc., impulse magnetizer (Model IM-10) and measured using the CTF cryogenic magnetometer. Sixteen pilot samples were given an IRM at $1.0 \mathrm{~T}$ and $\mathrm{AF}$ demagnetized so the demagnetization of IRM could be compared with that of NRM and ARM.

After all magnetic analyses were performed, polished thin sections were made from 10 representative samples. The thin sections were studied using reflected light microscopy and representative sections were selected from electron microanalysis. Electron microprobe analyses were performed to determine the chemical composi- tion (major and trace elements) of the opaque minerals. Ulvöspinel content $(X)$ was calculated as follows:

$$
X=3 /(1+\mathrm{Fe} / \mathrm{Ti})
$$

where $\mathrm{Fe} / \mathrm{Ti}=\left(0.777 \mathrm{FeO}+0.696 \mathrm{Fe}_{2} \mathrm{O}_{3}\right) /\left(0.6 \mathrm{TiO}_{2}\right)$.

Opaque minerals were analyzed with a Cameca SX-50 automated electron microprobe in the TAMU Geology Department, using a finely focused $(\approx 1 \mu \mathrm{m})$, nonrastered, spot-fixed electron beam. During analysis, the beam current was $30 \mathrm{nA}$ at $15 \mathrm{kev}$ for thin sections from Hole $504 \mathrm{~B}$ and $10 \mathrm{nA}$ at $15 \mathrm{kev}$ for thin sections from Hole $896 \mathrm{~A}$, which contained larger oxide grains. For ilmenite, magnetite, and maghemite analyses, $\mathrm{Si}$ and $\mathrm{Ca}$ were each counted for $10 \mathrm{~s}$; Ti and $\mathrm{Fe}$ were each counted for $20 \mathrm{~s} ; \mathrm{V}, \mathrm{Ni}$, and $\mathrm{Zn}$ were each counted for $30 \mathrm{~s} ; \mathrm{Mn}$ and $\mathrm{Cr}$ were each counted for $40 \mathrm{~s} ; \mathrm{Mg}$ was counted for $100 \mathrm{~s}$; and $\mathrm{Al}$ was counted for $110 \mathrm{~s}$. For the titanite analyses, $\mathrm{Ca}$ and Ti were each counted for $20 \mathrm{~s}, \mathrm{Mn}$ and Fe were each counted for 60 $\mathrm{s}$, and $\mathrm{Mg}$ and $\mathrm{Si}$ were each counted for $80 \mathrm{~s}$. Natural mineral standards used were diopside, spinel, $\mathrm{FeTiO}_{3}$, chromite, ilmenite, spessartine, and gahnite. Metal standards used were nickel and vanadium. Titanomagnetite and titanomaghemite weight percentages were recalculated following the method of Carmichael (1967) on the basis of 4 oxygens using a BASIC program obtained from J. Allan. $\mathrm{Fe}^{3+}$ values were determined by charge balance. Calculations for ilmenite and titanite followed the Cameca program, which uses the Deer et al. (1962) formulas. Ilmenite analyses were normalized by 6 oxygens, and titanite analyses were normalized by 18 oxygens.

\section{RESULTS}

\section{Rock Magnetic Properties}

Table 1 summarizes the hysteresis parameters of the samples studied. In this sample set, coercivity of Hole 504B samples ranges from 0.9 to $13.7 \mathrm{mT}($ mean $=5.46 \mathrm{mT})$, coercivity of remanence ranges from 12.5 to $38.6 \mathrm{mT}$ (mean $=23.0 \mathrm{mT}$ ), the ratio $H_{C R} / H_{C}$ ranges from 2.8 to $15.70 \mathrm{mT}$ (mean $=8.31$ ). Saturation magnetization $\left(J_{S}\right)$ ranges from 1.17 to $66.2 \mathrm{mAm}^{2} / \mathrm{kg}$ (mean $=28.09 \mathrm{mAm}^{2} / \mathrm{kg}$ ), saturation remanence $\left(J_{R S}\right)$ ranges from 0.016 to $2.57 \mathrm{mAm}^{2} / \mathrm{kg}$ (mean $=0.77 \mathrm{mAm}^{2} / \mathrm{kg}$ ), and the ratio $J_{R S} / J_{S}$ ranges from 0.012 to 0.154 (mean $=0.06$ ). Coercivity of Hole 896A samples ranges from 0.04 to $9.07 \mathrm{mT}$ (mean $=3.33 \mathrm{mT})$, coercivity of remanence ranges from 7.24 to $26.8 \mathrm{mT}$ (mean $=13.6 \mathrm{mT}$ ), the ratio $H_{C R} / H_{C}$ ranges from 2.05 to $181 \mathrm{mT}$ (mean $=32.2$ ). Saturation magnetization $\left(J_{S}\right)$ ranges from 2.34 to $46.9 \mathrm{mAm}^{2} / \mathrm{kg}$ (mean $=13.9 \mathrm{mAm}^{2} / \mathrm{kg}$ ), saturation remanence $\left(J_{R S}\right)$ ranges from 0.012 to $9.2 \mathrm{mAm}^{2} / \mathrm{kg}$ (mean $=1.39$ $\mathrm{mAm}^{2} / \mathrm{kg}$ ), and the ratio $J_{R S} / J_{S}$ ranges from 0.012 to 0.154 (mean $=$ 0.076).

Additional rock magnetic data from Hole 504B samples in the study are presented in Table 2. NRM intensity ranges from 0.01 to $0.24 \mathrm{~A} / \mathrm{m}$ (mean $=0.09 \mathrm{~A} / \mathrm{m}$ ). IRM intensity acquired at $1.0 \mathrm{~T}$ ranges from 6.79 to $445.89 \mathrm{~A} / \mathrm{m}$ (mean $=155.9 \mathrm{~A} / \mathrm{m}$ ) and saturation $\mathrm{ARM}$ (SARM) ranges from 0.05 to $0.80 \mathrm{~A} / \mathrm{m}$ (mean $=0.37 \mathrm{~A} / \mathrm{m})$. Data from Hole 896A samples are presented in Table 3. NRM intensity ranges from 0.14 to $22.59 \mathrm{~A} / \mathrm{m}$ (mean $=5.06 \mathrm{~A} / \mathrm{m}$ ). Saturation IRM ranges from 95.59 to $451.95 \mathrm{~A} / \mathrm{m}($ mean $=261.77 \mathrm{~A} / \mathrm{m})$ and saturation $\mathrm{ARM}$ ranges from 0.47 to $7.31 \mathrm{~A} / \mathrm{m}$ (mean $=3.69 \mathrm{~A} / \mathrm{m}$ ).

Figure 4 illustrates representative $\mathrm{AF}$ demagnetization behavior of NRM, IRM, and ARM for Samples 148-504B-247R-1, 60-62 cm, and 252R-1, 33-35 cm, and for Samples 148-896A-8R-1, 74-76 cm, and $24 \mathrm{R}-4,84-86 \mathrm{~cm}$. Sample 148-504B-247R-1, 60-62 cm, is a sparsely phyric plagioclase-olivine diabase containing about $10 \%$ patchy groundmass alteration. Sample 148-504B-252R-1, 33-35 cm, is a moderately phyric plagioclase-olivine-clinopyroxene diabase. Samples 148-896A-8R-1, 74-76 cm, and 24R-4, 84-86 cm, are moderately phyric plagioclase-olivine basalt. In these, as in all samples 
Table 1. Hysteresis data from Holes 504B and 896A.

\begin{tabular}{|c|c|c|c|c|c|c|c|c|c|}
\hline $\begin{array}{l}\text { Core, section, } \\
\text { interval }(\mathrm{cm})\end{array}$ & $\begin{array}{l}\text { Depth } \\
\text { (mbsf) }\end{array}$ & Unit & Lith & $\begin{array}{c}H_{C} \\
(\mathrm{mT})\end{array}$ & $\begin{array}{c}H_{C R} \\
(\mathrm{mT})\end{array}$ & $H_{C K} / H_{C}$ & $\begin{array}{c}J_{S} \\
(\mathrm{mAm} / \mathrm{kg})\end{array}$ & $\underset{\left(\mathrm{mAm} \mathrm{m}^{2} / \mathrm{kg}\right)}{J_{\text {s }}}$ & $J_{R S} / J_{S}$ \\
\hline \multicolumn{10}{|l|}{ 148-504B- } \\
\hline $241 \mathrm{R}-1,115-117$ & 2017.65 & 276 & POC & 5.10 & 23.80 & 4.67 & 47.37 & 2.57 & 0.05 \\
\hline 247R-1, 14-18 & 2056.84 & 285 & PO & 0.90 & 12.50 & 13.89 & 40.10 & 0.02 & 0.01 \\
\hline $247 \mathrm{R}-1,60-62$ & 2057.30 & 285 & $\mathrm{PO}$ & 1.60 & 15.40 & 9.63 & 66.20 & 0.51 & 0.02 \\
\hline 249R-1, 81-84 & 2072.01 & 290 & $\mathrm{CP}$ & 0.93 & 14.60 & 15.70 & 8.39 & 0.10 & 0.01 \\
\hline $251 \mathrm{R}-1,17-22$ & 2090.07 & 293 & POC & 10.50 & 32.90 & 3.13 & 5.31 & 1.25 & 0.11 \\
\hline $252 \mathrm{R}-1,33-37$ & 2099.73 & 293 & POC & 13.70 & 38.60 & 2.82 & 1.17 & 0.18 & 0.15 \\
\hline $\operatorname{Max}$ & & & & 13.70 & 38.60 & 15.70 & 66.20 & 2.57 & 0.15 \\
\hline Min & & & & 0.90 & 12.50 & 2.82 & 1.17 & 0.02 & 0.01 \\
\hline Mean & & & & 5.46 & 22.97 & 8.31 & 28.09 & 0.77 & 0.06 \\
\hline SD & & & & 5.47 & 10.78 & 5.62 & 26.83 & 0.99 & 0.06 \\
\hline \multicolumn{10}{|l|}{$148-896 \mathrm{~A}-$} \\
\hline $8 \mathrm{R}-1,74-76$ & 257.84 & 14 & PO & 8.15 & 16.70 & 2.05 & 46.90 & 9.20 & 0.20 \\
\hline $11 R-1,91-93$ & 286.91 & 14 & PO & 6.01 & 17.20 & 2.86 & 5.84 & 0.71 & 0.12 \\
\hline $16 \mathrm{R}-2,81-83$ & 336.06 & 24 & OP & 0.04 & 7.24 & 181.00 & 9.13 & 0.01 & 0.00 \\
\hline $16 \mathrm{R}-2,117-120$ & 336.42 & 24 & OP & 0.43 & 9.82 & 22.84 & 13.30 & 0.01 & 0.01 \\
\hline $21 \mathrm{R}-3,29-31$ & 376.27 & 32 & $\mathrm{PO}$ & 9.07 & 26.80 & 2.95 & 2.34 & 0.46 & 0.20 \\
\hline $22 \mathrm{R}-2,64-66$ & 384.73 & 32 & PO & 1.91 & 13.10 & 6.86 & 6.68 & 0.37 & 0.06 \\
\hline $24 \mathrm{R}-4,84-86$ & 407.08 & 36 & PO & 0.29 & 7.39 & 25.40 & 15.30 & 0.11 & 0.01 \\
\hline $30 \mathrm{R}-1,134-136$ & 460.64 & 51 & OP & 0.77 & 10.60 & 13.73 & 11.70 & 0.22 & 0.02 \\
\hline $\operatorname{Max}$ & & & & 9.07 & 26.80 & 181.00 & 46.90 & 9.20 & 0.20 \\
\hline Min & & & & 0.04 & 7.24 & 2.05 & 2.34 & 0.01 & 0.00 \\
\hline Mean & & & & 3.33 & 13.61 & 32.21 & 13.90 & 1.39 & 0.08 \\
\hline SD & & & & 3.79 & 6.54 & 60.81 & 13.99 & 3.17 & 0.08 \\
\hline
\end{tabular}

Notes: $\mathrm{Max}=$ maximum, $\mathrm{Min}=$ minimum, $\mathrm{Lith}=$ lithology, $\mathrm{POC}=$ plagioclase-olivine-clinopyroxene diabase, $\mathrm{CP}=$ clinopyroxene-plagioclase phyric diabase, $\mathrm{PO}=$ plagioclase-olivine diabase, and $\mathrm{OP}=$ olivine-plagioclase diabase. Other abbreviations defined in the text.

for which demagnetization of NRM, ARM, and IRM were compared, ARM is more stable to AF demagnetization than is IRM. Median destructive field (MDF) values for NRMs from Hole 504B range from 7 to $60 \mathrm{mT}$, with a mean of $31 \mathrm{mT}$. Hole 896A samples had MDFs ranging from 5 to $50 \mathrm{mT}$ and averaged $16 \mathrm{mT}$. Thermally demagnetized samples from Hole 896A showed inversion to hematite at about $300^{\circ} \mathrm{C}$, characteristic of titanomaghemite.

Volume susceptibility from Hole 504B samples ranges from 0.0014 to 0.098 (mean $=0.032 \pm 0.029 \mathrm{SI})$ similar to the mean value of the dikes obtained during previous legs (0.016-0.018 SI units; Pariso and Johnson, 1991; Pariso et al., 1995). Volume susceptibility from Hole 896A samples ranges from 0.002 to 0.104 SI (mean = $0.027 \pm 0.025 \mathrm{SI})$.

\section{Magnetic Oxide Petrography and Chemistry}

Three samples from Hole 504B were analyzed using the electron microprobe. Major- and trace-element geochemical analyses are presented in Tables 4, 5, and 6. Letters appended to sample names in the tables refer to different grains within the same thin section. A backscattered electron image from Sample 148-504B-247R-1, 60-62 cm, is presented in Figure 5.

Seven samples from Hole $896 \mathrm{~A}$ were studied by electron microanalysis. Major- and trace-element geochemical analyses are presented in Table 7. Letters appended to sample names in the tables refer to different grains within the same thin section. Figures $6 \mathrm{~A}-\mathrm{E}$ are backscattered electron images of representative samples from Hole $896 \mathrm{~A}$ that illustrate the range of grain morphologies observed.

\section{DISCUSSION AND CONCLUSIONS}

\section{Hole 504B}

\section{NRM and Susceptibility}

The range of NRM intensities measured in this suite of samples is consistent with that reported in Pariso et al. (1995), which reflects a general slight increase with depth in the sheeted dike section. Shipboard measurements during Leg 148 and the results presented here
Table 2. Rock-magnetic data for Hole 504B samples.

\begin{tabular}{|c|c|c|c|c|c|c|c|c|}
\hline $\begin{array}{l}\text { Core, section, } \\
\text { interval }(\mathrm{cm})\end{array}$ & $\begin{array}{l}\text { Depth } \\
\text { (mbsf) }\end{array}$ & Unit & Lith & $\begin{array}{l}\mathrm{NRM} \\
(\mathrm{A} / \mathrm{m})\end{array}$ & MDF & $\begin{array}{l}\text { IRM } \\
(\mathrm{A} / \mathrm{m})\end{array}$ & $\begin{array}{l}\text { SARM } \\
(\mathrm{A} / \mathrm{m})\end{array}$ & $\begin{array}{c}\mathrm{K} \\
(\mathrm{SI})\end{array}$ \\
\hline \multicolumn{9}{|l|}{ 148-504B- } \\
\hline 24 & 2007.05 & 272 & $\mathrm{P}$ & 0.01 & 12 & 223.54 & 0.57 & 0.0181 \\
\hline $241 \mathrm{~F}$ & & 27 & POC & & & & & \\
\hline $241 R-1,115-117^{*}$ & 2017. & 27 & POC & 0.0 & 42 & & 0.12 & \\
\hline $246 \mathrm{R}-1,70-73^{*}$ & 2052.90 & 283 & P & 0.24 & & 132.67 & 0.57 & \\
\hline $247 \mathrm{R}-1,14-1$ & 2056.8 & 28 & $\mathrm{PO}$ & 0.0 & 37 & 81 & 0 & \\
\hline $\mathrm{R}-1,6$ & 2057.30 & 285 & $\mathrm{PO}$ & 0.1 & 12 & 317 & 0.80 & 0.0980 \\
\hline 249R1, 8-11 & 2071.2 & 289 & A & 0.24 & 15 & 165 & 0.43 & 0.0308 \\
\hline$-1,81-84$ & 2072.01 & 290 & PO & 0.08 & 25 & 122 & 0.27 & 0.0333 \\
\hline 109 & 208 & 292 & POC & 0.11 & 2 & 306 & 0.62 & 79 \\
\hline$-22^{*}$ & 2090. & 293 & POC & 0.04 & 45 & 11 & 0.13 & 0.0031 \\
\hline$\because 25.27$ & 2090.15 & 293 & POC & 0.09 & 40 & 160 & 0.43 & 0.0315 \\
\hline $12+3$ & & 29 & & 0. & 2 & & 0 & 91 \\
\hline & 209 & 29 & POC & 0. & 6 & 133 & 0.28 & 0.0273 \\
\hline$-1,1$ & 2099. & 293 & POC & 0.03 & 4 & 11.69 & 0.10 & 0.0016 \\
\hline $252 \mathrm{R}-1,33-37$ & 2099.73 & 293 & POC & 0.02 & 45 & & 0.05 & \\
\hline Max & & & & 0.2 & 60 & 445 & 0.80 & \\
\hline Min & & & & 0.01 & 7 & 6.79 & 0.05 & 0.0014 \\
\hline Mean & & & & 0.09 & 31 & 0 & 0.37 & \\
\hline SD & & & & 0.08 & 15 & 127.05 & 0.23 & 0.0288 \\
\hline
\end{tabular}

Notes: $\mathrm{Max}=$ maximum, Min $=$ minimum, $\mathrm{SD}=$ standard deviation, Lith. $=$ lithology, $\mathrm{P}$ $=$ plagioclase phyric diabase, $\mathrm{POC}=$ plagioclase-olivine-clinopyroxene diabase, $\mathrm{PO}$ $=$ plagioclase-olivine diabase, and $\mathrm{A}=$ aphyric diabase. $K=$ volume susceptibility. Asterisks indicate samples with NRM, ARM, and IRM values that were AF demagnetized and compared. Other abbreviations defined in the text.

are lower than those reported from earlier legs to Site 504: the mean shipboard NRM from Leg 148 samples was 0.48 A/m (Alt, Kinoshita, Stokking, et al., 1993) and that of samples in this study is $0.09 \mathrm{~A} /$ $\mathrm{m}$. Leg 137/140 shipboard measurements averaged $3.0 \mathrm{~A} / \mathrm{m}$ (Dick, Erzinger, Stokking, et al., 1993). The Leg $148 \mathrm{MDF}$ averaged $25 \mathrm{mT}$ (Alt, Kinoshita, Stokking, et al., 1993), average MDF for samples in this study is 31, and that of Leg $137 / 140$ samples was only $9.6 \mathrm{mT}$ (Dick, Erzinger, Stokking, et al., 1992). Leg 148 shipboard paleomagnetists attributed these variations to the presence of a drilling-induced remanence, which affected the Leg 137/140 samples but not the Leg 148 material because of differences between the bottom-hole drilling assemblies used for Legs 137/140 and 148, rather than to differences in in-situ rock magnetic properties. Leg 148 samples are thus more representative of the actual in-situ values than are Leg 137/ 
Table 3. Rock-magnetic data for Hole 896A samples.

\begin{tabular}{|c|c|c|c|c|c|c|c|c|c|c|}
\hline $\begin{array}{l}\text { Core, section, } \\
\text { interval }(\mathrm{cm})\end{array}$ & $\begin{array}{l}\text { Depth } \\
\text { (mbsf) }\end{array}$ & Unit & Lith & Structure & $\% \mathrm{Ox}$ & $\begin{array}{l}\mathrm{NRM} \\
(\mathrm{A} / \mathrm{m})\end{array}$ & $\begin{array}{l}\text { MDF } \\
(\mathrm{mT})\end{array}$ & $\begin{array}{c}\mathrm{K} \\
(\mathrm{SI})\end{array}$ & $\begin{array}{l}\text { SARM } \\
(\mathrm{A} / \mathrm{m})\end{array}$ & $\begin{array}{l}\text { IRM } \\
(\mathrm{A} / \mathrm{m})\end{array}$ \\
\hline \multicolumn{11}{|l|}{$148-896 \mathrm{~A}-$} \\
\hline $2 \mathrm{R}-1,5-12$ & 200.95 & 4 & OP & Massive & 100 & 5.86 & 20 & 0.0067 & 3.26 & 253.53 \\
\hline $2 \mathrm{R}-1,32-38$ & 201.22 & 5 & OP & Massive & 100 & 9.25 & 40 & 0.0124 & 6.08 & 246.39 \\
\hline $4 \mathrm{R}-1,106-112$ & 219.96 & 10 & PO & Pillow & 100 & 4.33 & TH & 0.0192 & & \\
\hline $8 \mathrm{R}-1,74-76^{*}$ & 257.84 & 14 & PO & Pillow & 95 & 11.52 & 20 & 0.0020 & & 288.02 \\
\hline $10 \mathrm{R}-1,111-113$ & 277.51 & 14 & PO & Pillow & 35 & 22.59 & & 0.0036 & 6.27 & 332.95 \\
\hline $11 \mathrm{R}-1,15-17$ & 286.15 & 14 & PO & Pillow & 70 & 10.34 & 27 & 0.0160 & 5.35 & 372.40 \\
\hline $11 \mathrm{R}-1,91-93$ & 286.91 & 14 & PO & Pillow & 0 & 13.80 & & 0.0054 & 3.28 & 279.76 \\
\hline $12 \mathrm{R}-2,19-22$ & 297.24 & 16 & OP & Pillow/massive & 50 & 7.35 & 45 & 0.0076 & 0.47 & 317.68 \\
\hline $14 \mathrm{R}-3,61-63$ & 318.09 & 21 & OP & Pillow/massive & 100 & 8.55 & 15 & 0.0179 & 3.97 & 231.97 \\
\hline $15 \mathrm{R}-2,19-21$ & 325.88 & 23 & OP & Pillow & 0 & 11.50 & 32 & 0.0142 & 5.22 & 339.12 \\
\hline $16 \mathrm{R}-\mathrm{I}, 118-120$ & 335.08 & 24 & OP & Massive & 35 & 5.82 & 10 & 0.0420 & 3.12 & 256.88 \\
\hline 16R-2, 81-83* & 336.06 & 24 & OP & Massive & 60 & 4.68 & 10 & 0.0351 & 3.74 & 294.85 \\
\hline $16 \mathrm{R}-2,117-120^{*}$ & 336.42 & 24 & OP & Massive & 15 & 7.25 & 5 & 0.0285 & 3.78 & 270.56 \\
\hline $16 \mathrm{R}-3,51-53^{*}$ & 337.21 & 24 & OP & Massive & 60 & 4.56 & 5 & 0.0288 & 3.86 & 268.92 \\
\hline $16 \mathrm{R}-3,122-124$ & 337.92 & 24 & OP & Pillow & 0 & 15.26 & 17 & 0.0165 & 6.37 & 374.58 \\
\hline $17 \mathrm{R}-3,26-28$ & 346.7 & 27 & OP & Pillow & 50 & 3.91 & 15 & 0.0233 & 2.55 & 255.82 \\
\hline $20 \mathrm{R}-1,118-120$ & 364.08 & 31 & PO & Massive & 90 & 2.45 & 12 & 0.0203 & 4.72 & 344.58 \\
\hline $20 \mathrm{R}-1,142-144$ & 364.92 & 31 & PO & Massive & 95 & 2.94 & 12 & 0.0143 & 4.13 & 325.09 \\
\hline $21 \mathrm{R}-3,29-31 *$ & 376.27 & 32 & PO & Massive & 0 & 8.76 & 27 & 0.0129 & 7.31 & 451.95 \\
\hline $22 \mathrm{R}-1,0-5$ & 382.6 & 32 & PO & Massive & 10 & 7.10 & 17 & 0.0184 & 5.88 & 404.48 \\
\hline $22 \mathrm{R}-2,64-66^{*}$ & 384.73 & 32 & PO & Massive & 50 & 2.33 & 15 & 0.0157 & 4.57 & 337.07 \\
\hline $22 \mathrm{R}-3,129-131$ & 386.88 & 33 & PO & Massive & 60 & 9.91 & 10 & 0.0219 & 5.10 & 403.69 \\
\hline $23 \mathrm{R}-3,42-44$ & 395.42 & 36 & PO & Massive & 100 & 0.39 & 5 & 0.0335 & 1.48 & 110.35 \\
\hline $24 \mathrm{R}-1,8-10$ & 401.88 & 36 & PO & Massive & 7 & 2.59 & TH & 0.0718 & & \\
\hline $24 \mathrm{R}-1,111-113$ & 402.91 & 36 & PO & Massive & 0 & 0.68 & 5 & 0.0271 & 2.18 & 95.59 \\
\hline $24 \mathrm{R}-2,15-17$ & 403.45 & 36 & PO & Massive & 85 & 1.06 & 5 & 0.0108 & 2.32 & 194.29 \\
\hline $24 \mathrm{R}-3,32-35$ & 405.1 & 36 & PO & Massive & 90 & 1.61 & TH & 0.0585 & & \\
\hline $24 \mathrm{R}-3,94-96^{*}$ & 405.72 & 36 & PO & Massive & 100 & 1.58 & 5 & 0.1037 & 2.32 & 140.22 \\
\hline $24 R-4,63-65$ & 406.87 & 36 & PO & Massive & 75 & 1.53 & 5 & 0.0687 & 2.80 & 212.07 \\
\hline $24 \mathrm{R}-4,84-86^{*}$ & 407.08 & 36 & PO & Massive & 80 & 0.67 & 5 & 0.0115 & 3.25 & 163.00 \\
\hline $24 \mathrm{R}-5,45-47$ & 408.13 & 36 & PO & Massive & 85 & 1.28 & 7 & 0.0134 & 2.82 & 258.72 \\
\hline $24 \mathrm{R}-5,77-79$ & 408.45 & 36 & PO & Massive & 100 & 2.94 & 7 & 0.0902 & 3.06 & 261.28 \\
\hline $24 \mathrm{R}-5,125-127$ & 408.93 & 36 & PO & Massive & 98 & 3.24 & 7 & 0.0094 & 2.30 & 188.18 \\
\hline $25 \mathrm{R}-1,124-126$ & 412.54 & 37 & PO & Pillow & 60 & 1.64 & 20 & 0.0095 & 2.65 & 231.02 \\
\hline $26 \mathrm{R}-1,74-76$ & 421.74 & 38 & OP & Massive & 98 & 2.56 & 12 & 0.0075 & 3.59 & 270.79 \\
\hline $27 \mathrm{R}-1,41-44$ & 430.91 & 42 & OP & Breccia & 5 & 0.47 & 35 & 0.0117 & 4.00 & 118.16 \\
\hline $27 \mathrm{R}-1,52-54$ & 431.02 & 42 & OP & Massive & 0 & 1.55 & 50 & 0.0130 & 3.70 & 224.19 \\
\hline $27 \mathrm{R}-1,55-57$ & 431.05 & 42 & OP & Breccia & 0 & 0.14 & 25 & 0.0195 & 2.45 & 146.21 \\
\hline $27 \mathrm{R}-3,29-31$ & 433.66 & 46 & PO & Pillow & 5 & 2.08 & 15 & 0.0501 & 3.53 & 220.18 \\
\hline $29 \mathrm{R}-1,115-117^{*}$ & 450.85 & 49 & OP & Massive & 85 & 0.90 & 5 & 0.0274 & 2.36 & 175.14 \\
\hline $30 \mathrm{R}-1,134-136^{*}$ & 460.64 & 51 & OP & Pillow & 10 & 0.69 & 10 & 0.0934 & 2.86 & 287.60 \\
\hline Max & & & & & & 22.59 & 50 & 0.1037 & 7.31 & 451.95 \\
\hline Min & & & & & & 0.14 & 5 & 0.0020 & 0.47 & 95.59 \\
\hline Mean & & & & & & 5.06 & 16 & 0.0272 & 3.69 & 261.77 \\
\hline SD & & & & & & 4.91 & 12 & 0.0254 & 1.48 & 85.37 \\
\hline
\end{tabular}

Notes: $\mathrm{Max}=$ maximum, $\mathrm{Min}=$ minimum, $\mathrm{SD}=$ standard deviation, Lith $=$ lithology, $\mathrm{OP}=$ olivine-plagioclase phyric basalt, $\mathrm{PO}=$ plagioclase-olivine phyric basalt, $\%$ Ox $=$ percentage of groundmass alteration (from "Alteration Log" in Alt, Kinoshita, Stokking, et al., 1993). $K=$ volume susceptibility. Asterisks indicate samples whose NRM, ARM, and IRM were AF demagnetized and compared. Other abbreviations defined in the text.

140 samples (Alt, Kinoshita, Stokking, et al., 1993). Differences between the average value of shipboard data and data presented in this study may reflect the smaller number of samples in this study and/or correction of remanence data in this study by actual (rather than assumed) sample volume.

Magnetic remanence (natural and laboratory-induced) and magnetic susceptibility reflect the concentration of magnetic carriers in the rock. Samples whose magnetic intensity is low also have low magnetic susceptibility (Table 2 ) and low saturation magnetization (Table 1), as discussed in Pariso et al. (1995) and implying that the low remanence and susceptibility values reflect a decrease in the amount of magnetite. This variation in concentration of magnetite in turn reflects hydrothermal alteration, which causes loss of iron and replacement of ilmenite by anatase and/or rutile (Pariso and Johnson, 1991; Pariso et al., 1995). As observed by Pariso and Johnson (1991) and Pariso et al. (1995), samples whose magnetic intensity and susceptibility are low show evidence of high degrees of hydrothermal alteration, whereas samples whose magnetic intensity and susceptibility are moderate to high are less altered. Thus, variability in the amount of magnetizable material produced by differing degrees of hydrothermal alteration of the samples results in the wide variation in rock magnetic properties observed in this suite of samples.

\section{IRM and $A R M$}

Studies of acquisition of isothermal remanent magnetization (IRM) and anhysteretic remanent magnetization (ARM) were performed on board ship during Leg 148 (Alt, Kinoshita, Stokking, et al., 1993). Shipboard samples saturated in applied fields ranging from 90 and $200 \mathrm{mT}$, consistent with magnetite and/or titanomagnetite as the predominant remanence carrier, with a minor amount of higher coercivity minerals, such as hematite (Alt, Kinoshita, Stokking, et al., 1993). Shipboard ARMs were acquired in alternating fields up to 100 $\mathrm{mT}$, and in DC-biased fields of $0.032 \mathrm{mT}$, similar to that prevailing at Earth's field at Site 504 (Alt, Kinoshita, Stokking, et al., 1993). The shapes of ARM and IRM acquisition curves are consistent with the predominant magnetic carrier in Hole 504B being low-titanium magnetite.

\section{Magnetic Oxide Petrography and Chemistry}

The predominant magnetic oxide mineral in Hole 504B rocks recovered during Leg 148 is low-titanium magnetite, produced during high-temperature (greater than $500^{\circ}-600^{\circ} \mathrm{C}$ ) deuteric oxidation of primary titanomagnetite to magnetite plus ilmenite (Pariso and 

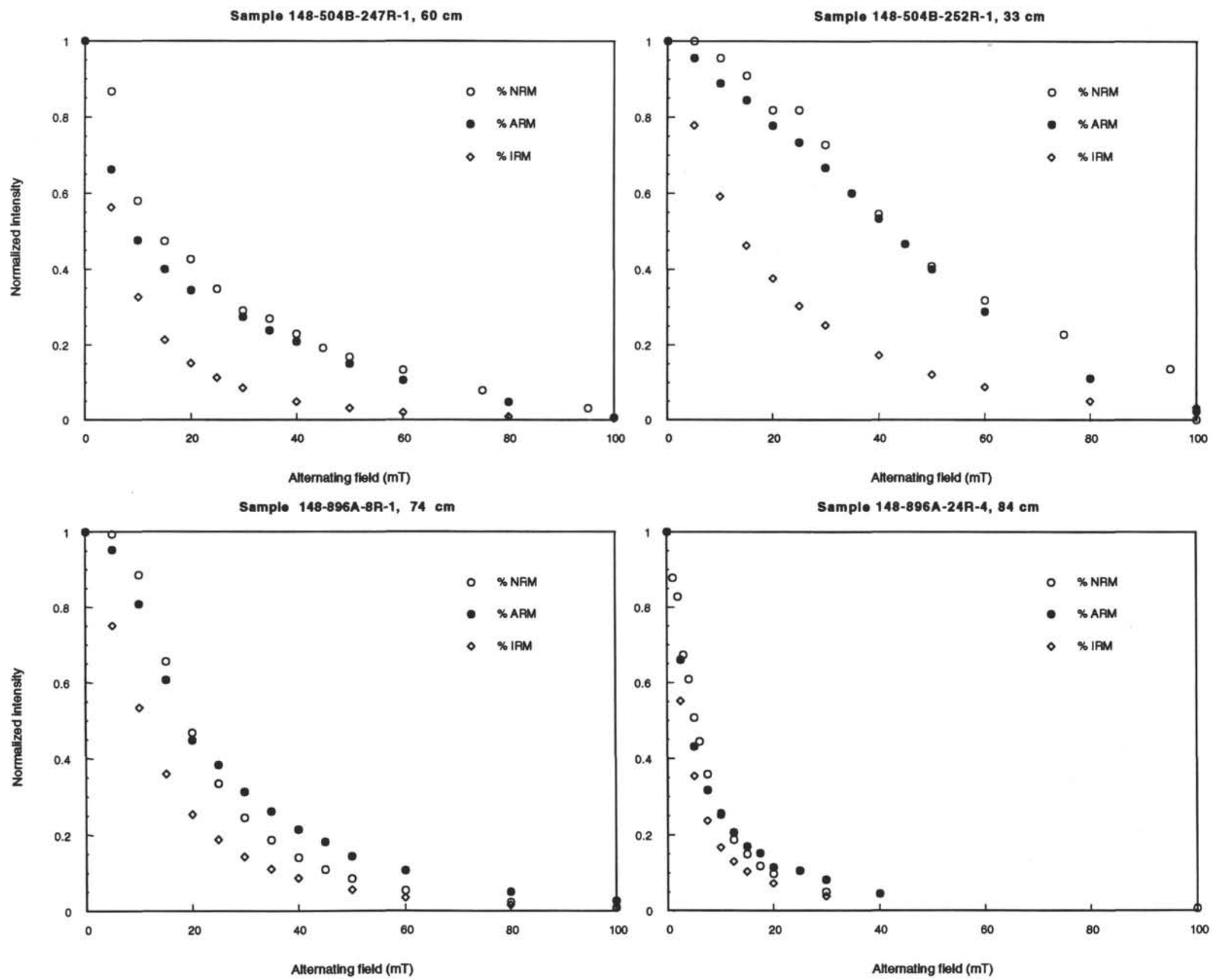

Figure 4. Alternating field (AF) demagnetization of NRM (open circles), ARM (filled circles), and IRM (diamonds) from representative samples. 
Table 4. Major- and trace-element analyses of magnetite lamellae from Hole 504B samples.

\begin{tabular}{|c|c|c|c|c|c|c|c|c|c|c|c|c|c|c|c|}
\hline $\begin{array}{l}\text { Core, section, } \\
\text { interval }(\mathrm{cm})\end{array}$ & $\mathrm{SiO}_{2}$ & $\mathrm{TiO}_{2}$ & $\mathrm{Al}_{2} \mathrm{O}_{3}$ & $\mathrm{~V}_{2} \mathrm{O}_{3}$ & $\mathrm{Cr}_{2} \mathrm{O}_{3}$ & $\mathrm{MnO}$ & $\mathrm{NiO}$ & $\mathrm{ZnO}$ & $\mathrm{MgO}$ & $\mathrm{CaO}$ & $\mathrm{FeO}$ & $\mathrm{Fe}_{2} \mathrm{O}_{3}$ & Total & $\mathrm{Fe} / \mathrm{Ti}$ & $X$ \\
\hline 148-504B- & & & & & & & & & & & & & & & \\
\hline $246 \mathrm{R}-1,70-73 \mathrm{a}$ & 0.055 & 0.683 & 0.019 & 0.706 & 0.089 & 0.029 & 0.048 & 0.051 & 0.003 & 0.301 & 31.612 & 67.502 & 101.098 & 174.582 & 0.017 \\
\hline $246 R-1,70-73 b$ & 0.111 & 1.145 & 0.040 & 0.464 & 0.049 & 0.029 & 0.000 & 0.017 & 0.031 & 0.171 & 32.301 & 66.727 & 101.085 & 104.134 & 0.029 \\
\hline $246 \mathrm{R}-1,70-73 \mathrm{c}$ & 0.133 & 2.114 & 0.729 & 0.712 & 0.069 & 0.082 & 0.002 & 0.000 & 0.005 & 0.115 & 33.292 & 63.403 & 100.656 & 55.185 & 0.053 \\
\hline $246 \mathrm{R}-1,70-73 \mathrm{c}$ & 0.095 & 2.325 & 0.337 & 0.759 & 0.083 & 0.091 & 0.021 & 0.163 & 0.008 & 0.035 & 33.534 & 64.014 & 101.465 & 50.616 & 0.058 \\
\hline $247 \mathrm{R}-1,60-62 \mathrm{a}$ & 0.083 & 1.950 & 1.134 & 1.221 & 0.135 & 0.037 & 0.031 & 0.094 & 0.004 & 0.072 & 33.423 & 63.389 & 101.573 & 59.905 & 0.049 \\
\hline $247 \mathrm{R}-1,60-62 \mathrm{a}$ & 0.201 & 2.226 & 0.192 & 0.887 & 0.066 & 0.047 & 0.048 & 0.047 & 0.023 & 0.071 & 33.612 & 64.020 & 101.440 & 52.916 & 0.056 \\
\hline $247 \mathrm{R}-1,60-62 \mathrm{~b}$ & 0.115 & 1.808 & 1.237 & 0.792 & 0.066 & 0.074 & 0.000 & 0.000 & 0.008 & 0.092 & 33.068 & 63.269 & 100.529 & 64.278 & 0.046 \\
\hline $247 \mathrm{R}-1,60-62 \mathrm{~b}$ & 0.083 & 1.514 & 0.913 & 0.852 & 0.088 & 0.044 & 0.017 & 0.000 & 0.014 & 0.021 & 32.903 & 64.459 & 100.908 & 77.531 & 0.038 \\
\hline $247 R-1,60-62 c$ & 0.091 & 2.252 & 0.489 & 0.423 & 0.040 & 0.112 & 0.000 & 0.012 & 0.003 & 0.088 & 32.968 & 63.072 & 99.550 & 51.446 & 0.057 \\
\hline $\operatorname{Max}$ & 0.201 & 2.325 & 1.237 & 1.221 & 0.135 & 0.112 & 0.048 & 0.163 & 0.031 & 0.301 & 33.612 & 67.502 & 101.573 & 174.582 & 0.058 \\
\hline Min & 0.055 & 0.683 & 0.019 & 0.423 & 0.040 & 0.029 & 0.000 & 0.000 & 0.003 & 0.021 & 31.612 & 63.072 & 99.550 & 50.616 & 0.017 \\
\hline Mean & 0.107 & 1.780 & 0.566 & 0.757 & 0.076 & 0.061 & 0.019 & 0.043 & 0.011 & 0.107 & 32.968 & 64.428 & 100.923 & 76.733 & 0.045 \\
\hline SD & 0.042 & 0.563 & 0.460 & 0.236 & 0.028 & 0.030 & 0.020 & 0.055 & 0.010 & 0.085 & 0.647 & 1.596 & 0.628 & 40.493 & 0.014 \\
\hline
\end{tabular}

Notes: $\mathrm{Max}=$ maximum, $\mathrm{Min}=$ minimum, and $\mathrm{SD}=$ standard deviation. Value $X$ (ulvöspinel content) explained in text. Lowercase letters appended to centimeter intervals refer to different grains within the same thin section.

Table 5. Major- and trace-element analyses of ilmenite lamellae from Hole 504B samples.

\begin{tabular}{|c|c|c|c|c|c|c|c|c|c|c|c|c|c|c|}
\hline $\begin{array}{l}\text { Core, section, } \\
\text { interval }(\mathrm{cm})\end{array}$ & $\mathrm{V}_{2} \mathrm{O}_{5}$ & $\mathrm{SiO}_{2}$ & $\mathrm{TiO}_{2}$ & $\mathrm{Al}_{2} \mathrm{O}_{3}$ & $\mathrm{Cr}_{2} \mathrm{O}_{3}$ & $\mathrm{Fe}_{2} \mathrm{O}_{3}$ & $\mathrm{MgO}$ & $\mathrm{CaO}$ & $\mathrm{MnO}$ & $\mathrm{FeO}$ & $\mathrm{NiO}$ & $\mathrm{ZnO}$ & Total & $\mathrm{Fe} / \mathrm{Ti}$ \\
\hline \multicolumn{15}{|l|}{ 148-504B- } \\
\hline $246 \mathrm{R}-1,70-73 \mathrm{a}$ & 0.5555 & 0.0443 & 44.5170 & 0.0337 & 0.0319 & 13.8480 & 0.0080 & 0.2809 & 1.3272 & 38.7112 & 0.0162 & 0.0000 & 99.3739 & 1.4870 \\
\hline $246 \mathrm{R}-1,70-73 b$ & 0.4127 & 0.0142 & 46.2403 & 0.0117 & 0.0129 & 11.8759 & 0.0365 & 0.2292 & 1.8072 & 39.6993 & 0.0046 & 0.0175 & 100.3619 & 1.4097 \\
\hline $246 \mathrm{R}-1,70-73 \mathrm{~b}$ & 0.4701 & 2.6309 & 45.6242 & 0.0874 & 0.0239 & 8.9141 & 0.0878 & 1.1944 & 1.6197 & 39.3021 & 0.0000 & 0.0000 & 99.9546 & 1.3422 \\
\hline $246 \mathrm{R}-1,70-73 \mathrm{c}$ & 0.4727 & 0.0675 & 47.4400 & 0.0044 & 0.0357 & 9.7348 & 0.0276 & 0.2599 & 1.7500 & 40.8574 & 0.0000 & 0.0512 & 100.7012 & 1.3533 \\
\hline $246 \mathrm{R}-1,70-73 \mathrm{c}$ & 0.4995 & 0.0046 & 47.0008 & 0.0049 & 0.0186 & 9.8896 & 0.0306 & 0.2852 & 1.7672 & 40.4320 & 0.0039 & 0.0000 & 99.9367 & 1.3581 \\
\hline $247 \mathrm{R}-1,60-62 \mathrm{a}$ & 0.7132 & 0.0726 & 47.2611 & 0.3818 & 0.0381 & 9.2879 & 0.1170 & 0.0198 & 1.9069 & 40.6454 & 0.0362 & 0.0100 & 100.4900 & 1.3417 \\
\hline $247 \mathrm{R}-1,60-62 \mathrm{a}$ & 0.8977 & 0.1750 & 47.8786 & 0.2739 & 0.0398 & 7.6430 & 0.0943 & 0.0533 & 2.2703 & 40.7978 & 0.0355 & 0.0100 & 100.1691 & 1.2887 \\
\hline $247 \mathrm{R}-1,60-62 \mathrm{~b}$ & 0.4021 & 0.8306 & 45.0026 & 0.1421 & 0.0241 & 12.0883 & 0.1225 & 0.7190 & 2.0756 & 38.2583 & 0.0000 & 0.0000 & 99.6652 & 1.4125 \\
\hline $247 \mathrm{R}-1,60-62 \mathrm{~b}$ & 0.6195 & 0.0895 & 47.1153 & 0.3555 & 0.0604 & 9.5331 & 0.1306 & 0.0097 & 1.9989 & 40.3889 & 0.0000 & 0.0088 & 100.3100 & 1.3448 \\
\hline $247 \mathrm{R}-1,60-62 \mathrm{c}$ & 0.4650 & 0.0501 & 47.5357 & 0.0405 & 0.0154 & 7.4159 & 0.0744 & 0.1843 & 1.9019 & 40.7221 & 0.0000 & 0.0364 & 98.4417 & 1.2903 \\
\hline $252 \mathrm{R}-1,33-37 \mathrm{a}$ & 0.3103 & 0.0667 & 47.0616 & 0.0103 & 0.0285 & 8.4845 & 0.0781 & 0.4969 & 2.0994 & 40.0741 & 0.0720 & 0.0000 & 98.7823 & 1.3119 \\
\hline $252 \mathrm{R}-1,33-37 \mathrm{a}$ & 0.3359 & 0.0670 & 49.5790 & 0.0051 & 0.0061 & 4.7270 & 0.0775 & 0.4300 & 1.9821 & 42.4435 & 0.0560 & 0.0838 & 99.7930 & 1.2192 \\
\hline $252 \mathrm{R}-1,33-37 \mathrm{a}$ & 0.2965 & 0.1306 & 47.2409 & 0.0308 & 0.0046 & 8.2555 & 0.0969 & 0.5622 & 1.9826 & 40.3232 & 0.0546 & 0.0200 & 98.9985 & 1.3081 \\
\hline $252 \mathrm{R}-1,33-37 \mathrm{~b}$ & 0.3224 & 0.0245 & 47.4451 & 0.0093 & 0.0000 & 8.6131 & 0.0484 & 0.3535 & 2.6089 & 39.9417 & 0.0068 & 0.0300 & 99.4038 & 1.3008 \\
\hline $252 \mathrm{R}-\mathrm{l}, 33-37 \mathrm{~b}$ & 0.3055 & 0.0358 & 47.1006 & 0.0089 & 0.0100 & 8.7942 & 0.0391 & 0.4259 & 2.2703 & 39.9957 & 0.0227 & 0.0488 & 99.0576 & 1.3162 \\
\hline $252 \mathrm{R}-1,33-37 \mathrm{~b}$ & 0.2790 & 0.0707 & 47.3762 & 0.0128 & 0.0107 & 8.2104 & 0.0426 & 0.3813 & 2.4169 & 40.0919 & 0.0000 & 0.0063 & 98.8989 & 1.2969 \\
\hline $252 \mathrm{R}-1,33-37 \mathrm{c}$ & 0.3929 & 0.3509 & 47.8589 & 0.0901 & 0.0235 & 8.0119 & 0.1370 & 0.4437 & 2.1547 & 40.6839 & 0.0417 & 0.0000 & 100.1891 & 1.2950 \\
\hline $252 \mathrm{R}-1,33-37 \mathrm{c}$ & 0.4580 & 0.1589 & 48.4793 & 0.0382 & 0.0370 & 6.5244 & 0.0717 & 0.5532 & 1.9968 & 41.4879 & 0.0492 & 0.0038 & 99.8585 & 1.2644 \\
\hline Max & 0.8977 & 2.6309 & 49.5790 & 0.3818 & 0.0604 & 13.8480 & 0.1370 & 1.19 & 2.6089 & 42.4435 & 0.0720 & 0.0838 & 100.7012 & 1.4870 \\
\hline Min & 0.2790 & 0.0046 & 44.5170 & 0.0044 & 0.0000 & 4.7270 & 0.0080 & 0.0097 & 1.3272 & 38.2583 & 0.0000 & 0.0000 & 98.4417 & 1.2192 \\
\hline Mean & 0.4560 & 0.2714 & 47.0976 & 0.0856 & 0.0234 & 8.9918 & 0.0734 & 0.3 & 1.9965 & 40.2698 & 0.0222 & 0.0 & 99.6881 & 1.7 ( \\
\hline SD & 0.1612 & 0.6193 & 1.18262 & 0.1229 & 0.0154 & 2.091522 & 0.03849 & 0.2799 & 0.2971 & 0.95126 & 0.0243 & 0.02347 & 0.651486 & 0.0612 \\
\hline
\end{tabular}

Notes: $\mathrm{Max}=$ maximum, $\mathrm{Min}=$ minimum, and $\mathrm{SD}=$ standard deviation. Lowercase letters appended to centimeter intervals refer to different grains within the same thin section.

Table 6. Major- and trace-element analyses of titanite from Hole 504B samples.

\begin{tabular}{lrllllll}
\hline $\begin{array}{l}\text { Core, section, } \\
\text { interval }(\mathrm{cm})\end{array}$ & $\mathrm{SiO}_{2}$ & $\mathrm{MgO}$ & $\mathrm{CaO}$ & $\mathrm{MnO}$ & $\mathrm{TiO}_{2}$ & $\mathrm{FeO}$ & Total \\
\hline 148-504B- & & & & & & & \\
246R-1, 70-73a & 38.114 & 4.527 & 21.565 & 0.087 & 23.383 & 6.321 & 93.997 \\
252R-1,33-37a & 30.396 & 0.211 & 28.125 & 0.041 & 32.608 & 2.056 & 93.437 \\
252R-1,33-37a & 30.462 & 0.130 & 28.274 & 0.029 & 34.618 & 1.167 & 94.680 \\
252R-1,33-37a & 31.410 & 0.577 & 27.424 & 0.039 & 32.688 & 1.439 & 93.577 \\
252R-1,33-37b & 31.029 & 0.504 & 27.486 & 0.000 & 29.278 & 1.888 & 90.185 \\
252R-1, 33-37b & 26.556 & 0.048 & 22.971 & 0.043 & 45.375 & 1.795 & 96.788 \\
252R-1,33-37c & 30.273 & 0.012 & 28.075 & 0.048 & 36.608 & 1.136 & 96.152 \\
252R-1,33-37c & 30.594 & 0.040 & 28.416 & 0.008 & 33.901 & 1.355 & 94.314 \\
252R-1,33-37c & 30.420 & 0.009 & 28.627 & 0.008 & 34.754 & 0.972 & 94.790 \\
252R-1,33-37c & 30.578 & 0.032 & 28.389 & 0.041 & 36.507 & 0.963 & 96.510 \\
252R-1,33-37c & 30.128 & 0.036 & 28.170 & 0.064 & 36.596 & 1.094 & 96.088 \\
Max & 38.114 & 4.527 & 28.627 & 0.087 & 45.375 & 6.321 & 96.788 \\
Min & 26.556 & 0.009 & 21.565 & 0.000 & 23.383 & 0.963 & 90.185 \\
Mean & 30.905 & 0.557 & 27.047 & 0.037 & 34.211 & 1.835 & 94.593 \\
SD & 2.703 & 1.332 & 2.412 & 0.026 & 5.370 & 1.535 & 1.882 \\
\hline
\end{tabular}

Note: $\mathrm{Max}=$ maximum, $\mathrm{Min}=$ minimum, and $\mathrm{SD}=$ standard deviation. Lowercase letters appended to centimeter intervals refer to different grains within the same thin section.
Johnson, 1991; Pariso et al., 1995). Subsequently, Leg 148 rocks were hydrothermally altered to varying extent: some or all ilmenite lamellae were replaced by titanite and/or a high-titanium mineral (anatase or rutile) and some iron was lost.

In all Hole 504B samples examined, high-temperature oxidation of primary titanomagnetite has produced lamellae or pods of magnetite and ilmenite. Hydrothermal alteration has further altered the minerals to a mixture of magnetite, ilmenite, titanite, and a hightitanium mineral (either rutile or anatase), as discussed in Pariso et al. (1995). Sample 148-504B-246R-1, 70-73 cm, is a sparsely phyric plagioclase-olivine diabase. Hydrothermal alteration of magnetoilmenite intergrowths falls in classes $\mathrm{H} 1$ and $\mathrm{H} 2$, and oxidation stage is $\mathrm{C} 3$. Intergrowths grains range up to $50 \mu \mathrm{m}$ in diameter. Sample $148-504 \mathrm{~B}-247 \mathrm{R}-1,60-62 \mathrm{~cm}$, is a moderately phyric plagioclaseolivine diabase exhibiting $\mathrm{H} 3$ hydrothermal alteration and $\mathrm{C} 2-\mathrm{C} 3$ oxidation. Intergrowths are up to $80 \mu \mathrm{m}$ in diameter. Sample 148$504 \mathrm{~B}-252 \mathrm{R}-1,33-37 \mathrm{~cm}$, is a moderately phyric plagioclase-olivineclinopyroxene diabase exhibiting $\mathrm{H} 4$ to $\mathrm{H} 5$ hydrothermal alteration and $\mathrm{C} 4$ oxidation.

Table 4 shows that magnetite lamellae tend to be enriched in trivalent elements: $\mathrm{Cr}_{2} \mathrm{O}_{3}$ averages $0.076 \mathrm{wt} \%$ in magnetite relative to $0.023 \mathrm{wt} \%$ in ilmenite, $\mathrm{Al}_{2} \mathrm{O}_{3}$ averages $0.566 \mathrm{wt} \%$ in magnetite and 
only $0.086 \mathrm{wt} \%$ in ilmenite, and $\mathrm{V}_{2} \mathrm{O}_{3}$ averages $0.757 \mathrm{wt} \%$ in magnetite whereas $\mathrm{V}_{2} \mathrm{O}_{5}$ averages $0.456 \mathrm{wt} \%$ in ilmenite. Divalent elements are partitioned into ilmenite lamellae: $\mathrm{MnO}$ averages $2.0 \mathrm{wt} \%$ in ilmenite and $0.0631 \mathrm{wt} \%$ in magnetite, and $\mathrm{MgO}$ averages $0.073 \mathrm{wt} \%$ in ilmenite and only $0.011 \mathrm{wt} \%$ in magnetite. These trends are consistent with those reported for sheeted dike samples from Legs 137 and 140 (Stokking et al., 1995).

Deer et al. (1962) and Haggerty (1976) report the partitioning of divalent ions ( $\mathrm{Mn}$ and $\mathrm{Mg}$ ) into ilmenite and trivalent ions $(\mathrm{Cr}, \mathrm{Al}, \mathrm{V})$ into magnetite when the two minerals are associated. This distribution was observed in altered and unaltered magnetite and in primary and secondary ilmenite from Hole 504B extrusives and upper sheeted dikes drilled during ODP Leg 83 (Kempton et al., 1985): $\mathrm{Al}_{2} \mathrm{O}_{3}$ and $\mathrm{Cr}_{2} \mathrm{O}_{3}$ concentrations are higher in both altered and unaltered magnetite than in ilmenite, whereas $\mathrm{MgO}$ and $\mathrm{MnO}$ are higher in both primary and secondary ilmenite than in associated magnetite or titanomagnetite. Vanadium concentrations were not reported. Thus, the variations in major and trace elements observed in the lower sheeted dikes drilled during Legs 137, 140, and 148 are consistent with trends observed in magnetite-ilmenite associations in general, as well as in the extrusives and upper sheeted dikes from Hole 504B.

\section{Particle Size and Magnetic Domain State}

Differences in the stabilities to AF demagnetization of natural and artificial remanences carried by magnetite are governed by particle size, the relative proportions of low- and high-coercivity particles, the degree of particle alignment, and the interplay between internal stresses within a particle and magnetostatic interactions between pinned and unpinned domain walls (Heider et al., 1992; Moon and Merrill, 1986; Stacey and Banerjee, 1974; Xu and Merrill, 1990; Xu and Dunlop, 1993). Lowrie and Fuller (1971) demonstrated that the relative stabilities to $\mathrm{AF}$ demagnetization of IRM and thermoremanent magnetization (TRM) may be used to evaluate the domain state that predominates in the primary carrier of remanence in a sample, assuming that the primary magnetization is carried by magnetite. For single-domain particles, TRM is more stable than IRM, whereas for multidomain particles, IRM is more stable than TRM. The stability of ARM to AF demagnetization is similar to that of TRM (Johnson et al., 1975; Levi and Merrill, 1976), which enabled the Lowrie-Fuller test to be modified to compare stabilities of ARM and IRM, thereby avoiding problems associated with mineralogical changes caused by heating the samples.

For equidimensional grains, pure magnetite is superparamagnetic when less than about $0.03 \mu \mathrm{m}$ in diameter, single domain from 0.03 to $0.10 \mu \mathrm{m}$ in diameter, and typically exhibits multidomain at diameters greater than $0.2 \mu \mathrm{m}$ (Butler and Banerjee, 1975). Particles larger than typical single-domain grains that show single-domain behavior are considered pseudo-single domain. The single domain threshold increases with Ti content. In addition, domain behavior is affected by the elongation of the particle (Butler and Banerjee, 1975), the structure of the grain, oxidation, or microcracks (Haggerty, 1976; Johnson and Hall, 1978; Henshaw and Merrill, 1980) that may produce regions within a large grain that act as single domains.

The ratio of hysteresis parameters $J_{R S} / J_{S}$ has been used to distinguish between single-domain and multidomain behaviors: values greater than 0.5 indicate single-domain particles, ratios between about 0.1 and 0.5 represent pseudo-single domain particles, and values less than about 0.1 indicate multidomain or superparamagnetic particles (Stoner and Wohlfarth, 1948; Day et al., 1977; Thompson and Oldfield, 1986). Although domain state is not a direct measure of particle size, the ratios may provide some indication of relative changes in domain behavior that reflect changes in particle size.

Samples for which stability to AF demagnetization of ARM and IRM were compared are indicated by an asterisk in Table 2 . In all samples, ARM is more stable to demagnetization than IRM, which

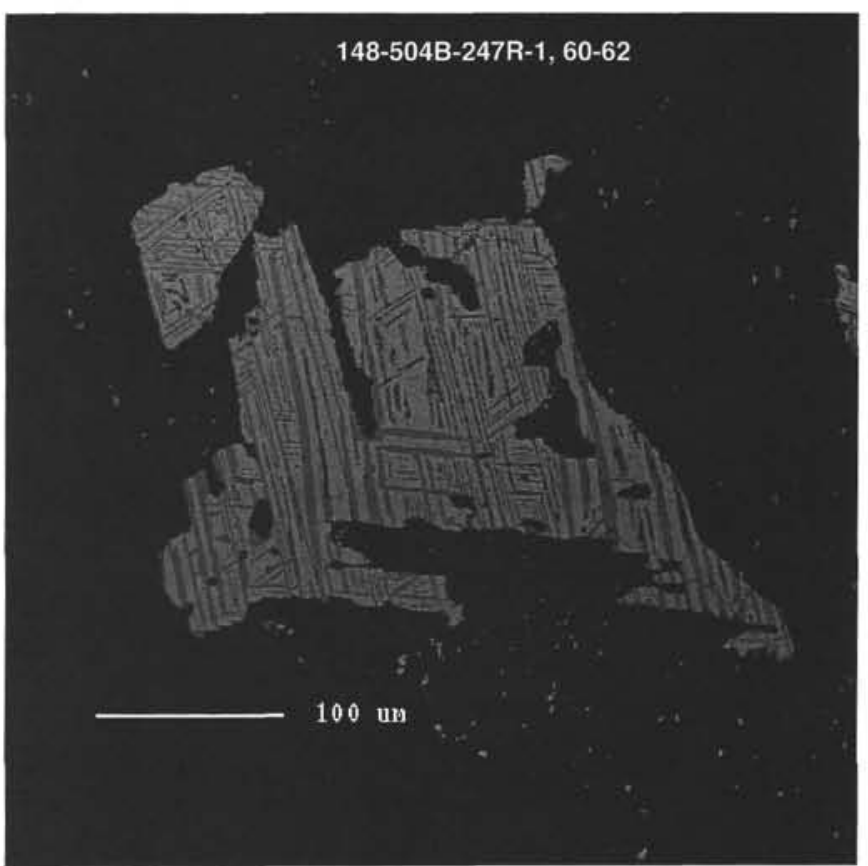

Figure 5. Backscattered electron image of Sample 148-504B-247R-1, 60-62 $\mathrm{cm}$. Dark bands represent ilmenite lamellae and lighter colored bands represent magnetite lamellae.

would indicate that the remanence carrier is single domain. However, the Lowrie-Fuller test, designed for pure equidimensional magnetite, may not be valid for magnetite exsolution lamellae relatively enriched in $\mathrm{V}_{2} \mathrm{O}_{3}, \mathrm{Al}_{2} \mathrm{O}_{3}$, and $\mathrm{Cr}_{2} \mathrm{O}_{3}$.

On the basis of hysteresis parameters, some samples from Hole 504B analyzed in this study exhibit either pseudo-single domain behavior, whereas other samples display behavior that is either multidomain or superparamagnetic (Table 1). Individual lamellae within the magnetite/ilmenite intergrowths observed by reflected light and electron microprobe analysis are thin, often less than one micron in width, and often broken into smaller regions by alteration to titanite or a high-titanium mineral. Thus, although the primary titanomagnetite grain may have been multidomain, after exsolution and subsequent hydrothermal alteration, the coherent magnetic domains have been reduced to the pseudo-single domain or superparamagnetic size regions.

\section{Hole 896A}

\section{NRM and Susceptibility}

Figure 7 illustrates the variation of NRM and susceptibility with depth in Hole 896A. In the upper part of the hole the intensity of natural remanence is high (shipboard samples: $11.9 \mathrm{~A} / \mathrm{m}$; this study: $8.18 \mathrm{~A} / \mathrm{m}$ ), the median destructive field is high (shipboard samples: $22.6 \mathrm{mT}$; this study: $18.6 \mathrm{mT}$ ), and the bulk susceptibility is relatively low (shipboard samples: $0.015 \mathrm{SI}$; this study: $0.017 \mathrm{SI}$ ). In the lower part of the hole, NRM intensity is lower (shipboard samples $=3.9$ $\mathrm{A} / \mathrm{m}$; this study $=1.45 \mathrm{~A} / \mathrm{m}$ ), median destructive fields are lower ( shipboard samples $=12.3 \mathrm{mT}$; this study $=13.1 \mathrm{mT}$ ), and bulk susceptibility values are higher (shipboard samples $=0.033 \mathrm{SI}$; this study $=0.038 \mathrm{SI})$. Maximum susceptibility values occur in Units 3136 (365-407.7 mbsf), which are massive flows that contain large, euhedral titanomaghemites (Fig. 6B, C).

Furuta and Levi (1983) observed similar trends in NRM, MDF and susceptibility data in the Hole 504B extrusive sequence. The change in magnetic properties in Hole 504B occurred below Core 69- 
504B-9R, at 332.2 mbsf. Furuta and Levi (1983) attributed these trends to the predominance of pillow units above and massive flows below 332.2 mbsf.

\section{IRM and ARM}

In both pillows and massive units from Hole 896A, shipboard isothermal remanent magnetism (IRM) acquisition studies indicate that the rocks generally saturate in applied fields between 70 and $200 \mathrm{mT}$, consistent with single-domain titanomagnetite and/or titanomaghemite as the carrier of remanence (Alt, Kinoshita, Stokking, et al., 1993). Some samples from massive flows saturated in fields greater than $200 \mathrm{mT}$, indicating the presence of a higher coercivity mineral, probably hematite (Alt, Kinoshita, Stokking, et al., 1993).

\section{Magnetic Oxide Petrography and Chemistry}

Low-temperature oxidation of primary titanomagnetite produced titanomaghemite with shrinkage cracks which were observed in all thin sections from Hole 896A that were analyzed with the electron microprobe. When plotted on a ternary diagram $\left(\mathrm{FeO}, \mathrm{TiO}_{2}, 0.5\right.$ $\mathrm{Fe}_{2} \mathrm{O}_{3}$ ), weight percent data from all samples analyzed failed to fall on the $\mathrm{Fe}_{3} \mathrm{O}_{4}-\mathrm{Fe}_{2} \mathrm{TiO}_{4}$ join, thus are titanomaghemite (cation-deficient nonstoichiometric spinels; Waychunas, 1991). Groundmass oxidation percentages (Table 3) are from the "Alteration Log" developed by Leg 148 shipboard petrologists (Alt, Kinoshita, Stokking, et al., 1993). Two microprobed samples are from the upper section, in which pillows are predominant. Sample 148-896A-8R-1, 74$76 \mathrm{~cm}$, is a moderately phyric plagioclase-olivine pillow basalt. Titanomaghemite grains are cruciform and subhedral to euhedral, and are less than $5 \mu \mathrm{m}$. Total oxidation (yellow-orange-red) of groundmass is $95 \%$. Numerous shrinkage cracks are present and are spaced from $<1$ to $<5 \mu \mathrm{m}$ apart. Sample 148-896A-11R-1, 91-93 cm, is a moderately phyric plagioclase-olivine pillow basalt containing subhedral titanomaghemite up to $10 \mu \mathrm{m}$ in diameter. Shrinkage cracks divide the grains into regions $<1$ to $5 \mu \mathrm{m}$ in size.

Five microprobed samples are from the lower section, dominated by massive units. Sample 148-896A-24R-1, 111-113 cm, is a massive moderately phyric plagioclase-olivine basalt with subhedral titanomaghemite laths ranging up to $100 \times 40 \mu \mathrm{m}$ in size. Groundmass appears unoxidized; on board ship, the rock was described as gray and fresh-looking (Alt, Kinoshita, Stokking, et al., 1993). Shrinkage cracks are well developed in titanomaghemite and are spaced from 2 to $>10 \mu \mathrm{m}$ apart. Sample $148-896 \mathrm{~A}-24 \mathrm{R}-3,94-96 \mathrm{~cm}$, is a massive moderately phyric plagioclase-olivine basalt containing subhedral laths of titanomaghemite up to $40 \mu \mathrm{m}$ across. Abundant shrinkage cracks break the titanomaghemite grain into regions from $<1$ to $5 \mu \mathrm{m}$ across. Reddish oxidative alteration is extensive: oxidation of groundmass is $100 \%$. Sample $148-896 \mathrm{~A}-24 \mathrm{R}-4,84-86 \mathrm{~cm}$, is a massive moderately phyric plagioclase-olivine basalt with cruciform and skeletal grains of maghemite up to $30 \mu \mathrm{m}$ in diameter. The sample contains red and red-brown oxidative halos. Total oxidation of groundmass is $80 \%$. Well-developed shrinkage cracks divide the grains into regions from $<1$ to $10 \mu \mathrm{m}$ across. Sample $148-896 \mathrm{~A}-29 \mathrm{R}-$ $1,115-117 \mathrm{~cm}$, is a moderately phyric olivine-plagioclase basalt with euhedral to subhedral maghemite laths up to $60 \mu \mathrm{m}$ in size. Total oxidation of groundmass is $85 \%$ and some reddish oxidative alteration halos are present. Shrinkage cracks are numerous and break the grains into regions ranging in size from $<1$ to $5 \mu \mathrm{m}$. Sample 148$896 \mathrm{~A}-30 \mathrm{R}-1,134-136 \mathrm{~cm}$, is a moderately phyric olivine-plagioclase pillow basalt containing cruciform maghemite less than $5 \mu \mathrm{m}$ in diameter. Total oxidation of groundmass is $10 \%$. Shrinkage cracks are abundant, well developed, and break the grain into submicronsized regions.

Ulvöspinel content (Table 7) in titanomaghemite ranges from about 0.217 to 0.647 , with a mean of 0.508 . The highest values are from Unit 36: the massive basalt containing large euhedral tita- nomaghemites (Fig. 6B, C). The lowest values are from pillow units in the upper part of Hole 896A.

Concentrations of $\mathrm{V}_{2} \mathrm{O}_{3}\left(0.185-1.227 \mathrm{wt} \%\right.$ ) and $\mathrm{Cr}_{2} \mathrm{O}_{3}$ (below detection limit to $0.131 \mathrm{wt} \%$, mean $=0.064 \mathrm{wt} \%$ ) are similar to those measured in magnetite lamellae from Hole 504B samples. Aluminum is relatively enriched in Hole 896A titanomaghemite, ranging from 0.807 to $3.586 \mathrm{wt} \%$ and averaging $1.697 \mathrm{wt} \%$. The concentration of $\mathrm{MnO}(0.153-3.367 \mathrm{wt} \%$; mean $=1.257 \mathrm{wt} \%)$ is similar to that in ilmenite lamellae in Hole 504B intergrowths; however, that of $\mathrm{MgO}$ is somewhat higher $(0.08-2.165 \mathrm{wt} \%$; mean $=0.577 \mathrm{wt} \%)$.

\section{Particle Size and Magnetic Domain State}

As for samples from Hole 504B, all samples from Hole 896A for which stability to AF demagnetization of ARM and IRM were compared exhibited behavior characteristic of single domain grains (Figure 4). Again, the Lowrie-Fuller test may not be valid: the carrier of remanence in Hole 896A samples is titanomaghemite, relatively enriched in several trivalent and divalent cations, rather than pure magnetite. Grain morphologies vary from cruciform to euhedral but are rarely equidimensional.

Hysteresis parameters determined from Hole 896A are highly variable, reflecting the range of grain sizes and morphologies and possibly the density and abundance of shrinkage cracks as well. Samples from pillow units (Unit 14: Samples 148-896A-8R-1, 74-76 cm, and 11R-1, 91-93 cm; Unit 51: Sample 148-896A-30R-1, 134-136 $\mathrm{cm}$ ) fall within the range characteristic of either superparamagnetic or multidomain behavior. The cruciform habit and abundant and dense shrinkage cracks of titanomaghemite within the pillow units make it unlikely that the predominant carrier is multidomain. Many submicron regions were observed during electron microanalysis, indicating the presence of at least some superparamagnetic material (Fig. 6E).

Samples from the massive basalt of Unit 36 also exhibit behavior that may either be superparamagnetic or multidomain. Titanomaghemite grains in this unit are large and euhedral and contain relatively few shrinkage cracks (Fig. 6B, C); hence, they are probably multidomain.

\section{CONCLUSIONS}

Samples of lowermost sheeted dikes from Hole 504B recovered during Leg 148 contain low-titanium magnetite, produced during high-temperature deuteric oxidation of primary titanomagnetite to intergrowths of magnetite and ilmenite. Varying degrees of hydrothermal alteration has replaced some or all ilmenite lamellae by titanite and/or a high-titanium mineral (anatase or rutile) and some iron has been lost.

The variability in the amount of magnetizable material produced by differing degrees of hydrothermal alteration of the samples of lowermost sheeted dikes from Hole 504B results in the wide variation in rock magnetic properties observed in this suite of samples, as observed by Pariso and Johnson (1991) and Pariso et al. (1995) in upper portions of the sheeted dike complex.

The ratio of saturation remanence to saturation magnetization in samples of lowermost sheeted dikes from Hole 504B is consistent with pseudo-single-domain behavior.

Magnetite lamellae in Hole 504B magnetite/ilmenite intergrowths from lowermost sheeted dikes are enriched in the trivalent oxides $\mathrm{Cr}_{2} \mathrm{O}_{3}$ (mean $\left.=0.076 \mathrm{wt} \%\right), \mathrm{Al}_{2} \mathrm{O}_{3}$ (mean $=0.566 \mathrm{wt} \%$ ), and $\mathrm{V}_{2} \mathrm{O}_{3}$ (mean $=0.757 \mathrm{wt} \%$ ), whereas divalent oxides are concentrated in ilmenite lamellae (mean $\mathrm{MnO}=1.997 \mathrm{wt} \%$ and mean $\mathrm{MgO}=0.073$ wt $\%)$.

The primary carrier of remanence in volcanic samples recovered from Hole $896 \mathrm{~A}$ is titanomaghemite produced by low-temperature oxidation of primary titanomagnetite. 
Table 7. Major- and trace-element analyses of titanomaghemite in Hole 896A samples.

\begin{tabular}{|c|c|c|c|c|c|c|c|c|}
\hline $\begin{array}{l}\text { Core, section, } \\
\text { interval (cm) }\end{array}$ & $\mathrm{SiO}_{2}$ & $\mathrm{TiO}_{2}$ & $\mathrm{Al}_{2} \mathrm{O}_{3}$ & $\mathrm{~V}_{2} \mathrm{O}_{3}$ & $\mathrm{Cr}_{2} \mathrm{O}_{3}$ & $\mathrm{MnO}$ & $\mathrm{NiO}$ & $\mathrm{ZnO}$ \\
\hline $148-896 \mathrm{~A}-$ & & & & & & & & \\
\hline $8 \mathrm{R}-1,74-76 \mathrm{a}$ & 0.167 & 9.674 & 3.586 & 0.674 & 0.063 & 0.397 & 0.000 & 0.129 \\
\hline $8 \mathrm{R}-1,74-76 \mathrm{a}$ & 0.430 & 9.527 & 3.506 & 0.661 & 0.075 & 0.377 & 0.000 & 0.091 \\
\hline $8 \mathrm{R}-1,74-76 \mathrm{a}$ & 0.287 & 11.228 & 3.050 & 0.532 & 0.059 & 0.452 & 0.046 & 0.194 \\
\hline $8 \mathrm{R}-1,74-76 \mathrm{~b}$ & 0.134 & 12.018 & 2.784 & 0.475 & 0.039 & 0.494 & 0.021 & 0.019 \\
\hline $8 \mathrm{R}-1,74-76 \mathrm{~b}$ & 0.188 & 12.522 & 3.041 & 0.455 & 0.075 & 0.447 & 0.055 & 0.107 \\
\hline $8 \mathrm{R}-1,74-76 \mathrm{c}$ & 0.299 & 17.285 & 2.694 & 0.515 & 0.051 & 0.539 & 0.000 & 0.141 \\
\hline $8 \mathrm{R}-1,74-76 \mathrm{~d}$ & 0.149 & 11.303 & 3.068 & 0.630 & 0.096 & 0.549 & 0.002 & 0.148 \\
\hline $11 R-1,91-93 a$ & 0.159 & 10.078 & 1.149 & 0.602 & 0.037 & 0.284 & 0.042 & 0.076 \\
\hline $11 \mathrm{R}-1,91-93 \mathrm{~b}$ & 0.086 & 11.823 & 1.883 & 0.672 & 0.055 & 0.227 & 0.081 & 0.046 \\
\hline $11 \mathrm{R}-1,91-93 \mathrm{~b}$ & 0.097 & 10.934 & 2.019 & 0.815 & 0.110 & 0.219 & 0.044 & 0.042 \\
\hline $11 \mathrm{R}-1,91-93 \mathrm{~b}$ & 0.173 & 10.326 & 2.141 & 0.862 & 0.064 & 0.183 & 0.051 & 0.004 \\
\hline IIR-1,91-93b & 0.084 & 9.717 & 2.221 & 0.817 & 0.097 & 0.153 & 0.118 & 0.000 \\
\hline $11 \mathrm{R}-1,91-93 \mathrm{~b}$ & 0.110 & 9.404 & 2.199 & 0.853 & 0.099 & 0.224 & 0.083 & 0.091 \\
\hline $11 \mathrm{R}-1,91-93 \mathrm{c}$ & 0.132 & 8.122 & 1.958 & 0.878 & 0.103 & 0.217 & 0.118 & 0.000 \\
\hline $11 \mathrm{R}-1,91-93 \mathrm{c}$ & 0.195 & 9.172 & 2.053 & 0.934 & 0.110 & 0.197 & 0.000 & 0.049 \\
\hline $11 \mathrm{R}-1,91-93 \mathrm{c}$ & 0.161 & 9.182 & 2.264 & 0.992 & 0.131 & 0.186 & 0.000 & 0.000 \\
\hline $11 \mathrm{R}-1,91-93 \mathrm{c}$ & 0.166 & 10.306 & 1.726 & 0.675 & 0.076 & 0.173 & 0.002 & 0.141 \\
\hline $11 \mathrm{R}-1,91-93 \mathrm{~d}$ & 0.198 & 13.916 & 2.072 & 0.721 & 0.061 & 0.219 & 0.028 & 0.126 \\
\hline $11 \mathrm{R}-1,91-93 \mathrm{~d}$ & 0.111 & 11.999 & 2.225 & 0.898 & 0.094 & 0.242 & 0.095 & 0.228 \\
\hline $11 R-1,91-93 d$ & 0.125 & 13.693 & 2.079 & 0.715 & 0.083 & 0.226 & 0.009 & 0.034 \\
\hline IIR-1, $91-93 \mathrm{~d}$ & 0.255 & 13.857 & 2.073 & 0.765 & 0.073 & 0.207 & 0.042 & 0.057 \\
\hline $11 \mathrm{R}-1,91-93 \mathrm{~d}$ & 0.121 & 12.132 & 2.136 & 0.690 & 0.081 & 0.248 & 0.000 & 0.004 \\
\hline $24 \mathrm{R}-1,111-113 \mathrm{a}$ & 0.012 & 20.358 & 0.996 & 0.590 & 0.059 & 0.495 & 0.060 & 0.098 \\
\hline $24 \mathrm{R}-1,111-113 \mathrm{a}$ & 0.098 & 19.426 & 1.214 & 0.550 & 0.049 & 0.543 & 0.000 & 0.042 \\
\hline $24 \mathrm{R}-1,111-113 \mathrm{a}$ & 0.053 & 19.087 & 1.209 & 0.333 & 0.018 & 0.544 & 0.028 & 0.045 \\
\hline $24 \mathrm{R}-1,111-113 \mathrm{a}$ & 0.127 & 18.744 & 1.180 & 0.260 & 0.049 & 0.507 & 0.007 & 0.068 \\
\hline $24 \mathrm{R}-1,111-113 \mathrm{a}$ & 0.149 & 18.979 & 0.908 & 0.238 & 0.030 & 0.511 & 0.005 & 0.215 \\
\hline $24 \mathrm{R}-1,111-113 \mathrm{~b}$ & 0.124 & 20.706 & 1.160 & 0.611 & 0.045 & 0.506 & 0.092 & 0.098 \\
\hline $24 \mathrm{R}-1,111-113 \mathrm{~b}$ & 0.127 & 21.401 & 1.010 & 0.544 & 0.025 & 0.499 & 0.000 & 0.011 \\
\hline $24 \mathrm{R}-1,111-113 \mathrm{~b}$ & 0.092 & 20.822 & 1.150 & 0.628 & 0.084 & 0.508 & 0.005 & 0.083 \\
\hline $24 \mathrm{R}-1,111-113 \mathrm{~b}$ & 0.102 & 20.653 & 1.150 & 0.700 & 0.072 & 0.510 & 0.062 & 0.076 \\
\hline $24 \mathrm{R}-1,111-113 \mathrm{c}$ & 0.141 & 19.835 & 0.834 & 0.461 & 0.020 & 0.436 & 0.000 & 0.185 \\
\hline $24 \mathrm{R}-1,111-113 \mathrm{c}$ & 0.108 & 19.882 & 0.873 & 0.330 & 0. & 0. & 0.009 & 0. \\
\hline $24 \mathrm{R}-1,111-113 \mathrm{~d}$ & 0.078 & 20.024 & 0.950 & 0.185 & 0.039 & 0.547 & 0.000 & 0.166 \\
\hline $24 \mathrm{R}-1,111-113 \mathrm{~d}$ & 0.188 & 19.715 & 1.174 & 0.268 & 0.000 & 0.532 & 0.048 & 0.110 \\
\hline $24 \mathrm{R}-1,111-113 \mathrm{~d}$ & 0.113 & 20.048 & 1.085 & 0.321 & 0.005 & 0.569 & 0.021 & 0.083 \\
\hline $24 \mathrm{R}-1,111-113 \mathrm{~d}$ & 0.065 & 20.147 & 0.947 & 0.307 & 0.000 & 0.518 & 0.000 & 0.159 \\
\hline $24 \mathrm{R}-1,111-113 \mathrm{~d}$ & 0.224 & 19.800 & 0.997 & 0.279 & 0.030 & 0.530 & 0.041 & 0.019 \\
\hline $24 \mathrm{R}-1,111-113 \mathrm{e}$ & 0.149 & 20.317 & 1.047 & 0.472 & 0.020 & 0.459 & 0.000 & 0.110 \\
\hline $24 \mathrm{R}-1,111-113 \mathrm{e}$ & 0.146 & 20.428 & 1.256 & 0.429 & 0.049 & 0.512 & 0.129 & 0.045 \\
\hline $24 \mathrm{R}-1,111-113 \mathrm{e}$ & 0.059 & 20.326 & 1.362 & 0.654 & 0.085 & 0.489 & 0.000 & 0.000 \\
\hline $24 \mathrm{R}-1,111-113 \mathrm{e}$ & 0.094 & 20.303 & 1.280 & 0.669 & 0.084 & 0.469 & 0.030 & 0.155 \\
\hline $24 \mathrm{R}-1,111-113 \mathrm{e}$ & 0.153 & 19.916 & 1.242 & 0.730 & 0.047 & 0.586 & 0.034 & 0.253 \\
\hline $24 \mathrm{R}-3,94-96 \mathrm{a}$ & 0.319 & 22.277 & 1.674 & 0.962 & 0.117 & 2.444 & 0.000 & 0.221 \\
\hline $24 \mathrm{R}-3,94-96 \mathrm{a}$ & 0.145 & 22.958 & 1.709 & 0.970 & 0.082 & 2.508 & 0.000 & 0.141 \\
\hline $24 \mathrm{R}-3,94-96 \mathrm{a}$ & 0.175 & 23.081 & 1.704 & 1.032 & 0.089 & 2.593 & 0.044 & 0.141 \\
\hline $24 \mathrm{R}-3,94-96 \mathrm{a}$ & 0.343 & 22.511 & 1.762 & 0.954 & 0.076 & 2.647 & 0.032 & 0.156 \\
\hline $24 \mathrm{R}-3,94-96 \mathrm{a}$ & 0.131 & 22.890 & 1.401 & 0.813 & 0.080 & 2.583 & 0.016 & 0.327 \\
\hline $24 \mathrm{R}-3,94-96 \mathrm{~b}$ & 0.194 & 22.209 & 1.627 & 0.880 & 0.064 & 2.442 & 0.030 & 0.217 \\
\hline $24 \mathrm{R}-3,94-96 \mathrm{~b}$ & 0.149 & 22.336 & 1.522 & 0.794 & 0.076 & 2.528 & 0.000 & 0.103 \\
\hline $24 \mathrm{R}-3,94-96 \mathrm{~b}$ & 0.852 & 22.365 & 1.676 & 0.660 & 0.100 & 2.416 & 0.032 & 0.137 \\
\hline $24 \mathrm{R}-3,94-96 \mathrm{~b}$ & 0.126 & 21.766 & 1.260 & 0.593 & 0.061 & 2.162 & 0.000 & 0.106 \\
\hline $24 \mathrm{R}-3,94-96 \mathrm{c}$ & 0.133 & 23.443 & 1.690 & 0.623 & 0.079 & 2.424 & 0.035 & 0.213 \\
\hline $24 \mathrm{R}-3,94-96 \mathrm{c}$ & 0.236 & 23.208 & 1.922 & 0.904 & 0.057 & 2.299 & 0.0 & $0.1 \quad 5$ \\
\hline $24 \mathrm{R}-3,94-96 \mathrm{c}$ & 0.086 & 22.997 & 2.141 & 0.908 & 0.114 & 1.535 & 0.000 & 0.194 \\
\hline $24 \mathrm{R}-3,94-96 \mathrm{c}$ & 0.115 & 21.857 & 2.508 & 0.977 & 0.104 & 0.927 & 0.058 & 0.198 \\
\hline $24 \mathrm{R}-3,94-96 \mathrm{c}$ & 0.324 & 20.666 & 2.943 & 1.227 & 0.097 & 1.066 & 0.062 & 0.145 \\
\hline $24 \mathrm{R}-3,94-96 \mathrm{~d}$ & 0.317 & 23.208 & 1.522 & 0.845 & 0.078 & 2.438 & 0.000 & 0.281 \\
\hline $24 \mathrm{R}-3,94-96 \mathrm{~d}$ & 0.265 & 23.240 & 1.67 & 0.7 & 0.0 & 2.592 & 0.037 & 0.194 \\
\hline $24 \mathrm{R}-3,94-96 \mathrm{~d}$ & 0.122 & 23.668 & 1.4 & & & 2.5 & & \\
\hline $24 \mathrm{R}-3$ & 0.458 & 22.747 & 1.492 & 0.586 & 0.103 & 2.550 & 0.053 & 0.103 \\
\hline $24 \mathrm{R}-3,94-96 \mathrm{~d}$ & 0.211 & 23.237 & i.168 & 0.641 & 0.073 & 2.648 & 0.02 & 0.175 \\
\hline $24 \mathrm{R}-3,94-96 \mathrm{e}$ & 0.186 & 21.908 & 1.5 & 0.4 & 0. & 2.3 & 0.0 & 0.217 \\
\hline$-3,94-96$ & 0.253 & 22.006 & 1.4 & 0.6 & 0.0 & 2.3 & 0.0 & 0.080 \\
\hline$-3,94-96 \mathrm{e}$ & 0.178 & 21.787 & 1.38 & 0.6 & 0.0 & 2.13 & 0.0 & 0.3 \\
\hline $24 \mathrm{R}-3,94-96 \mathrm{e}$ & 0.252 & 21.575 & 1.328 & 0.6 & 0.05 & 2.400 & 0.4 & 0.065 \\
\hline $24 \mathrm{R}-3,94-96 \mathrm{e}$ & 0.135 & 21.794 & $\begin{array}{l}1.603 \\
\text {. }\end{array}$ & 0.553 & 0.066 & 2.072 & 0.01 & 0.175 \\
\hline$-3,94-96 \mathrm{f}$ & 0.075 & 22.057 & 1.5 & 0. & 0. & 2.3 & 0. & 0.1 \\
\hline $3,94-96 \mathrm{f}$ & 0. & 22.4 & 1.591 & 0.8 & 0. & 2.6 & 0.0 & 0.2 \\
\hline $3,94-9$ & 0.069 & 22.103 & 1.517 & 0.9 & 0. & 2.4 & 0.0 & 0.3 \\
\hline $24 \mathrm{R}-3,94-96 \mathrm{f}$ & 0.389 & 21.429 & 1.326 & & & 2.3 & & 0.1 \\
\hline $24 \mathrm{R}-3,94-96 \mathrm{f}$ & 0.387 & 22.236 & $\begin{array}{l}1.684 \\
.620\end{array}$ & 0.820 & 0 & 2.190 & 0.0 & 0.107 \\
\hline $3,94-96 \mathrm{~g}$ & 0.099 & 22.262 & 1.4 & 0.658 & 0.085 & 2.270 & 0.00 & 0.076 \\
\hline $94-96 \mathrm{~g}$ & 0. & 21.7 & 1.5 & 0.4 & 0. & 2.2 & 0.0 & 0.2 \\
\hline$-3,94-96 \mathrm{~g}$ & 0.160 & 20.982 & 1.371 & 0.47 & 0.0 & 1.862 & 0.0 & 0.255 \\
\hline $24 \mathrm{R}-3,94-96 \mathrm{~h}$ & 0.173 & 21.411 & 2.173 & & & 1.252 & 0.0 & 0.1 \\
\hline $24 \mathrm{R}-3,94-96 \mathrm{~h}$ & 0.275 & 20.994 & 2.11 & 1. & 0. & 1.553 & 0.053 & 0.129 \\
\hline $\mathrm{R}-3,94-96 \mathrm{i}$ & 0.162 & 21.573 & 1.43 & 0.6 & 0.0 & 2.285 & 0.051 & 0.160 \\
\hline 3 & 0. & 22. & 1.6 & 0.7 & 0.0 & 1.949 & 0.002 & 0.175 \\
\hline $3,94-96 \mathrm{i}$ & 0.248 & 22.553 & 1.624 & 0.635 & 0.06 & 2.521 & 0.000 & 0.148 \\
\hline$-3,94-96 \mathrm{i}$ & 0.908 & 22.093 & 1.668 & 0.540 & 0.030 & 2.461 & 0.03 & 0.179 \\
\hline $24 \mathrm{R}-4,84-86 \mathrm{a}$ & 0.388 & 22.362 & 1.777 & 0.972 & 0.0 & 1.719 & 0.03 & 0.18 \\
\hline $4,84-86 a$ & 0.073 & 22.66 & 1.5 & 0. & 0. & 1.898 & 0.00 & 0.247 \\
\hline $4,84-86 a$ & & 22.5 & 1.5 & 0. & 0. & 1.8 & 0.0 & 0.100 \\
\hline 4,84 & 0.206 & 22.218 & 1.912 & 0.860 & 0.11 & 1.497 & 0.030 & 0.232 \\
\hline $24 \mathrm{R}-4,84-86 \mathrm{~b}$ & 0.126 & 19.519 & 2.676 & 1.207 & 0.089 & 0.606 & 0.113 & 0.000 \\
\hline $24 \mathrm{R}-4,84-86 \mathrm{~b}$ & 0.193 & 18.835 & 2.630 & 1.079 & 0.119 & 0.351 & 0.042 & 0.000 \\
\hline $24 \mathrm{R}-4,84-86 \mathrm{~b}$ & 0.171 & 18.000 & 2.585 & 1.074 & 0.060 & 0.300 & 0.000 & 0.091 \\
\hline
\end{tabular}


Table 7 (continued).

\begin{tabular}{|c|c|c|c|c|c|c|c|c|c|c|c|c|c|c|c|}
\hline $\begin{array}{l}\text { Core, section, } \\
\text { interval }(\mathrm{cm})\end{array}$ & $\mathrm{SiO}_{2}$ & $\mathrm{TiO}_{2}$ & $\mathrm{Al}_{2} \mathrm{O}_{3}$ & $\mathrm{~V}_{2} \mathrm{O}_{3}$ & $\mathrm{Cr}_{2} \mathrm{O}_{3}$ & $\mathrm{MnO}$ & $\mathrm{NiO}$ & $\mathrm{ZnO}$ & $\mathrm{MgO}$ & $\mathrm{CaO}$ & $\mathrm{FeO}$ & $\mathrm{Fe}_{2} \mathrm{O}_{3}$ & Total & $\mathrm{Fe} / \mathrm{Ti}$ & $X$ \\
\hline $24 R-4,84-86 b$ & 0.130 & 21.060 & 1.294 & 0.349 & 0.007 & 1.138 & 0.058 & 0.152 & 0.142 & 0.054 & 48.905 & 25.116 & 98.406 & 4.391 & 0.557 \\
\hline $24 \mathrm{R}-4,84-86 \mathrm{~b}$ & 0.390 & 20.326 & 1.099 & 0.213 & 0.031 & 1.743 & 0.065 & 0.179 & 0.110 & 0.138 & 47.586 & 25.705 & 97.586 & 4.499 & 0.546 \\
\hline $24 \mathrm{R}-4,84-86 \mathrm{~b}$ & 0.170 & 20.877 & 1.354 & 0.237 & 0.005 & 1.527 & 0.000 & 0.159 & 0.124 & 0.040 & 48.461 & 25.329 & 98.283 & 4.413 & 0.554 \\
\hline $24 R-4,84-86 c$ & 0.535 & 21.849 & 1.537 & 0.764 & 0.047 & 1.626 & 0.000 & 0.068 & 0.211 & 0.084 & 49.844 & 22.199 & 98.764 & 4.133 & 0.584 \\
\hline $24 \mathrm{R}-4,84-86 \mathrm{c}$ & 0.385 & 20.386 & 1.392 & 0.412 & 0.011 & 1.534 & 0.000 & 0.201 & 0.140 & 0.075 & 48.191 & 25.558 & 98.285 & 4.516 & 0.544 \\
\hline $24 R-4,84-86 c$ & 0.029 & 20.605 & 1.311 & 0.351 & 0.000 & 1.223 & 0.000 & 0.220 & 0.163 & 0.062 & 48.387 & 26.606 & 98.957 & 4.539 & 0.542 \\
\hline $24 R-4,84-86 c$ & 0.111 & 22.450 & 1.361 & 0.693 & 0.071 & 1.261 & 0.014 & 0.118 & 0.249 & 0.070 & 50.092 & 22.346 & 98.836 & 4.044 & 0.595 \\
\hline $24 R-4,84-86 c$ & 0.132 & 22.129 & 1.352 & 0.573 & 0.068 & 0.950 & 0.000 & 0.129 & 0.276 & 0.062 & 49.890 & 22.590 & 98.151 & 4.104 & 0.588 \\
\hline $24 \mathrm{R}-4,84-86 \mathrm{~d}$ & 0.150 & 22.442 & 1.921 & 0.710 & 0.067 & 1.335 & 0.000 & 0.179 & 0.401 & 0.146 & 49.576 & 21.207 & 98.133 & 3.957 & 0.605 \\
\hline $24 \mathrm{R}-4,84-86 \mathrm{~d}$ & 0.592 & 22.131 & 1.196 & 0.535 & 0.058 & 1.682 & 0.000 & 0.228 & 0.278 & 0.135 & 49.466 & 21.546 & 97.846 & 4.024 & 0.597 \\
\hline $24 R-4,84-86 d$ & 0.101 & 22.402 & 2.186 & 0.775 & 0.071 & 0.841 & 0.071 & 0.049 & 0.400 & 0.072 & 50.119 & 20.915 & 98.003 & 3.980 & 0.602 \\
\hline $29 \mathrm{R}-1,115-117 \mathrm{a}$ & 0.248 & 20.692 & 1.774 & 0.629 & 0.066 & 2.265 & 0.028 & 0.137 & 0.173 & 0.111 & 47.635 & 24.753 & 98.511 & 4.369 & 0.559 \\
\hline $29 \mathrm{R}-1.115-117 \mathrm{a}$ & 0.153 & 19.386 & 1.756 & 0.698 & 0.074 & 1.195 & 0.023 & 0.125 & 0.290 & 0.057 & 47.313 & 27.577 & 98.647 & 4.811 & 0.516 \\
\hline $29 \mathrm{R}-1,115-117 \mathrm{a}$ & 0.213 & 21.034 & 2.161 & 0.846 & 0.059 & 1.845 & 0.000 & 0.034 & 0.222 & 0.126 & 48.188 & 23.002 & 97.730 & 4.235 & 0.573 \\
\hline $29 \mathrm{R}-1,115-117 \mathrm{~b}$ & 0.081 & 18.076 & 1.622 & 0.541 & 0.053 & 0.941 & 0.048 & 0.068 & 0.631 & 0.068 & 45.701 & 30.763 & 98.594 & 5.248 & 0.480 \\
\hline $29 \mathrm{R}-1,115-117 \mathrm{~b}$ & 0.110 & 17.865 & 1.585 & 0.560 & 0.039 & 0.789 & 0.062 & 0.053 & 0.751 & 0.024 & 45.598 & 31.286 & 98.722 & 5.337 & 0.473 \\
\hline $29 \mathrm{R}-1,115-117 \mathrm{~b}$ & 0.889 & 17.134 & 2.001 & 0.989 & 0.090 & 0.951 & 0.051 & 0.042 & 0.957 & 0.123 & 45.299 & 29.657 & 98.182 & 5.432 & 0.466 \\
\hline $29 \mathrm{R}-1,115-117 \mathrm{c}$ & 0.124 & 14.643 & 1.554 & 0.719 & 0.080 & 0.398 & 0.046 & 0.023 & 0.697 & 0.032 & 43.426 & 37.934 & 99.676 & 6,846 & 0.382 \\
\hline $29 \mathrm{R}-1,115-117 \mathrm{c}$ & 0.045 & 14.043 & 1.743 & 0.684 & 0.091 & 0.413 & 0.060 & 0.049 & 0.877 & 0.033 & 42.463 & 39.173 & 99.675 & 7.152 & 0.368 \\
\hline $29 \mathrm{R}-1,115-117 \mathrm{c}$ & 0.169 & 14.463 & 1.400 & 0.650 & 0.042 & 0.741 & 0.000 & 0.034 & 0.401 & 0.059 & 43.090 & 37.623 & 98.673 & 6.876 & 0.381 \\
\hline $29 \mathrm{R}-1,115-117 \mathrm{c}$ & 0.216 & 16.003 & 0.807 & 0.640 & 0.058 & 0.735 & 0.076 & 0.034 & 0.293 & 0.037 & 44.613 & 35.184 & 98.696 & 6.161 & 0.419 \\
\hline $29 \mathrm{R}-1,115-117 \mathrm{c}$ & 0.237 & 13.987 & 1.728 & 0.610 & 0.074 & 0.500 & 0.055 & 0.042 & 0.757 & 0.086 & 42.473 & 38.366 & 98.915 & 7.114 & 0.370 \\
\hline $30 \mathrm{R}-1,134-136 \mathrm{a}$ & 0.650 & 19.901 & 1.777 & 0.559 & 0.048 & 3.367 & 0.051 & 0.179 & 0.189 & 0.527 & 44.983 & 23.834 & 96.065 & 4.316 & 0.564 \\
\hline $\operatorname{Max}$ & 0.908 & 23.668 & 3.586 & 1.227 & 0.131 & 3.367 & 0.129 & 0.375 & 2.165 & 0.527 & 50.285 & 49.999 & 101.649 & 12.809 & 0.647 \\
\hline Min & 0.012 & 8.122 & 0.807 & 0.185 & 0.000 & 0.153 & 0.000 & 0.000 & 0.080 & 0.000 & 35.549 & 18.729 & 96.065 & 3.640 & 0.217 \\
\hline Mean & 0.203 & 18.968 & 1.697 & 0.673 & 0.064 & 1.257 & 0.029 & 0.125 & 0.577 & 0.106 & 46.390 & 28.484 & 98.572 & 5.390 & 0.508 \\
\hline SD & 0.160 & 4.367 & 0.565 & 0.225 & 0.029 & 0.900 & 0.031 & 0.080 & 0.561 & 0.095 & 4.177 & 8.745 & 1.270 & 2.156 & 0.120 \\
\hline
\end{tabular}

Notes: Max = maximum, Min = minimum, and $\mathrm{SD}=$ standard deviation. Value $X$ (ulvöspinel content) explained in text. Lowercase letters appended to centimeter intervals refer to different grains within the same thin section.

The Hole $896 \mathrm{~A}$ interval may be divided into two zones on the basis of magnetic properties: the section above $390 \mathrm{mbsf}$, predominantly pillow basalts, is characterized by relatively high NRM intensities, low magnetic susceptibilities, and particle sizes that range from the superparamagnetic to pseudosingle domain regions. Massive units in the section below 390 mbsf exhibit lower NRM intensities, higher magnetic susceptibilities, resulting from magnetic particle sizes that range from single to multidomain.

Ulvöspinel content in titanomaghemites from Hole 896A averages 0.508 , and ranges from 0.217 to 0.647 . Titanomaghemite appears slightly enriched in $\mathrm{Al}_{2} \mathrm{O}_{3}$ (mean $=1.697 \mathrm{wt} \%$ ), $\mathrm{Cr}_{2} \mathrm{O}_{3}$ (mean $=0.064$ $\mathrm{wt} \%), \mathrm{V}_{2} \mathrm{O}_{3}$ (mean $\left.=0.673 \mathrm{wt} \%\right), \mathrm{MnO}(1.257 \mathrm{wt} \%)$, and $\mathrm{MgO}$ $(0.577 \mathrm{wt} \%)$.

\section{ACKNOWLEDGMENTS}

We thank J. Aguiar and C. Cooper for assistance with data processing. We are grateful to R. Guillemette for his guidance and assistance with the electron microprobe and to J. Allan and J. Gee for advice and assistance with microprobe data processing. We thank D. Merrill for his patient efforts in the TAMU Paleomagnetic Laboratory. We are indebted to R. Musgrave for performing the hysteresis measurements and to K. Verosub for kindly allowing us the use of his equipment at University of California, Davis. We thank Ram Alkaly, UCLA Thin Section Laboratory, for preparing polished thin sections of exceptionally high quality. Constructive reviews by J. Pariso, B. Hausen, J. Alt, and P. Michael considerably improved this manuscript. This research was supported by a grant to L.S. from the U.S. Science Support Program of the Joint Oceanographic Institutions, Inc.

\section{REFERENCES}

Ade-Hall, J.M., Khan, M.A., Dagley, P., and Wilson, R.L., 1968. A detailed opaque petrological and magnetic investigation of a single Tertiary lava flow from Skye, Scotland, I. Iron-titanium oxide petrology. Geophys. J. R. Astron. Soc., 16:375-388.
Ade-Hall, J.M., Palmer, H.C., and Hubbard, T.P., 1971. The magnetic and opaque petrological response of basalts to regional hydrothermal alteration. Geophys. J. R. Astron. Soc., 24:137-174.

Alt, J.C., Honnorez, J., Laverne, C., and Emmermann, R., 1986. Hydrothermal alteration of a $1 \mathrm{~km}$ section through the upper oceanic crust, Deep Sea Drilling Project Hole 504B: mineralogy, chemistry, and evolution of seawater-basalt interactions. J. Geophys. Res., 91:10309-10335.

Alt, J.C., Kinoshita, H., Stokking, L.B., et al., 1993. Proc. ODP, Init. Repts. 148: College Station, TX (Ocean Drilling Program).

Anderson, R.N., Honnorez, J., Becker, K., Adamson, A.C., Alt, J.C., Emmermann, R., Kempton, P.D., Kinoshita, H., Laverne, C., Mottl, M.J., and Newmark, R.L., 1982. DSDP Hole 504B, the first reference section over $1 \mathrm{~km}$ through Layer 2 of the oceanic crust. Nature, 300:589-594.

Anderson, R.N., Honnorez, J., Becker, K., et al., 1985. Init. Repts. DSDP, 83: Washington (U.S. Govt. Printing Office).

Becker, K., Foss, G., et al., 1992. Proc. ODP, Init. Repts., 137: College Station, TX (Ocean Drilling Program).

Becker, K., Sakai, H., Adamson, A.C., Alexandrovich, J., Alt, J.C., Anderson, R.N., Bideau, D., Gable, R., Herzig, P.M., Houghton, S.D., Ishizuka, H., Kawahata, H., Kinoshita, H., Langseth, M.G., Lovell, M.A., Malpas, J., Masuda, H., Merrill, R.B., Morin, R.H., Mottl, M.J., Pariso, J.E., Pezard, P.A., Phillips, J.D., Sparks, J.W., and Uhlig, S., 1989. Drilling deep into young oceanic crust, Hole 504B, Costa Rica Rift. Rev. Geophys., 27:79-102.

Becker, K., Sakai, H., et al., 1988. Proc. ODP, Init. Repts., 111: College Station, TX (Ocean Drilling Program).

Becker, K., Sakai, H., et al., 1989. Proc. ODP, Sci. Results, 111: College Station, TX (Ocean Drilling Program).

Butler, R.F., and Banerjee, S.K., 1975. Theoretical single-domain grain-size range in magnetite and titanomagnetite. J. Geophys. Res., 80:4049-4058.

Cande, S.C., and Kent, D.V., 1992. A new geomagnetic polarity time scale for the Late Cretaceous and Cenozoic. J. Geophys. Res., 97:1391713951.

Cann, J.R., Langseth, M.G., Honnorez, J., Von Herzen, R.P., White, S.M., et al., 1983. Init. Repts. DSDP, 69: Washington (U.S. Govt. Printing Office).

Carmichael, I.S.E., 1967. The iron-titanium oxides of salic volcanic rocks and their associated ferromagnesian silicates. Contrib. Mineral. Petrol. 14:36-64.

Cisowski, S., 1980. The relationship between the magnetic properties of terrestrial igneous rocks and the composition and internal structure of their component Fe-oxide grains. Geophys. J. R. Astron. Soc., 60:107-122. 
CRRUST (Costa Rica Rift United Scientific Team), 1982. Geothermal regimes of the Costa Rica Rift, east Pacific, investigated by drilling, DSDP-IPOD Legs 68, 69, and 70. Geol. Soc. Am. Bull., 93:862-875.

Day, R., Fuller, M., and Schmidt, V.A., 1977. Hysteresis properties of titanomagnetites: grain-size and compositional dependence. Phys. Earth Planet. Inter., 13:260-267.

Deer, W.A., Howie, R.A., and Zussman, J., 1962. Rock-forming Minerals (Vol. 5): Non-silicates: New York (Wiley).

Dick, H.J.B., Erzinger, J., Stokking, L.B., et al., 1992. Proc. ODP, Init. Repts., 140: College Station, TX (Ocean Drilling Program).

Dunlop, D.J., 1969. Hysteretic properties of synthetic and natural monodomain grains. Philos. Mag., 19:329-338.

Evans, M.E., and Wayman, M.L., 1974. An investigation of the role of ultrafine titanomagnetite intergrowths in paleomagnetism. Geophys. J. R. Astron. Soc., 36:1-10.

Furuta, T., and Levi, S., 1983. Basement paleomagnetism of Hole 504B. In Cann, J.R., Langseth, M.G., Honnorez, J., Von Herzen, R.P., White, S.M., et al., Init. Repts. DSDP, 69: Washington (U.S. Govt. Printing Office), 697-703.

Haggerty, S.E., 1976. Oxidation of opaque mineral oxides in basalts. In Rumble, D., III (Ed.), Oxide Minerals. Short Course Notes-Mineral. Soc. Am., 3:101-277.

, 1991. Oxide textures: a mini-atlas. In Lindsley, D.H. (Ed.), Oxide Minerals: Petrologic and Magnetic Significance. Rev. Mineral., 25:129219.

Hall, J.M., and Fisher, B.E., 1987. The characteristics and significance of secondary magnetite in a profile through the dike component of the Troodos, Cyprus, ophiolite. Can. J. Earth Sci., 24:2141-2159.

Heider, F., Dunlop, D.J., and Soffel, H.C., 1992. Low-temperature and alternating field demagnetization of saturation remanence and thermoremanence in magnetite grains $(0.037 \mu \mathrm{m}$ to $5 \mathrm{~mm})$. J. Geophys. Res. 97:9371-9381.

Henshaw, R.C., Jr., and Merrill, R.T., 1980. Magnetic and chemical changes in marine sediments. Rev. Geophys. Space Phys., 18:483-504.

Hobart, M.A., Langseth, M.G., and Anderson, R.N., 1985. A geothermal and geophysical survey on the south flank of the Costa Rica Rift: Sites 504 and 505. In Anderson, R.N., Honnorez, J., Becker, K., et al., Init. Repts. DSDP, 83: Washington (U.S. Govt. Printing Office), 379-404.

Johnson, H.P., and Hall, J.M., 1978. A detailed rock magnetic and opaque mineralogy study of the basalts from the Nazca Plate. Geophys. J. R. Astron. Soc., 52:45-64.

Johnson, H.P., Lowrie, W., and Kent, D.V., 1975. Stability of anhysteretic remanent magnetization in fine and coarse magnetite and maghemite particles. Geophys. J. R. Astron. Soc., 41:1-10.

Kempton, P.D., Autio, L.K., Rhodes, J.M., Holdaway, M.J., Dungan, M.A., and Johnson, P., 1985. Petrology of basalts from Hole 504B, Deep Sea Drilling Project, Leg 83. In Anderson, R.N., Honnorez, J., Becker, K., et al., Init. Repts. DSDP, 83: Washington (U.S. Govt. Printing Office), 129-164.

Leinen, M., Rea, D.K., et al., 1986. Init. Repts. DSDP, 92: Washington (U.S. Govt. Printing Office).
Levi, S., and Merrill, R.T., 1976. A comparison of ARM and TRM in magnetite. Earth Planet. Sci. Lett., 32:171-184.

Lowrie, W., and Fuller, M., 1971. On the alternating field demagnetization characteristics of multidomain thermoremanent magnetization in magnetite. J. Geophys. Res., 76:6339-6349.

Moon, T., and Merrill, R.T., 1986. Magnetic screening in multidomain material. J. Geomagn. Geoelectr., 38:883-894.

Pariso, J.E., and Johnson, H.P., 1991. Alteration processes at Deep Sea Drilling Project/Ocean Drilling Program Hole 504B at the Costa Rica Rift: implications for magnetization of oceanic crust. J. Geophys. Res., 96:11703-11722.

Pariso, J.E., Stokking, L., and Allerton, S., 1995. Rock magnetism and magnetic mineralogy of a 1-km section of sheeted dikes, Hole 504B. In Erzinger, J., Becker, K., Dick, H.J.B., and Stokking, L.B. (Eds.), Proc. ODP, Sci. Results, 137/140: College Station, TX (Ocean Drilling Program), 253-262.

Rice, P.D., Hall, J.M., and Opdyke, N.D., 1980. Deep Drill 1972: a paleomagnetic study of the Bermuda Seamount. Can. J. Earth Sci., 17:232243.

Smith, G.M., and Banerjee, S.K., 1986, Magnetic structure of the upper kilometer of the marine crust at Deep Sea Drilling Project Hole 504B, Eastern Pacific Ocean. J. Geophys. Res., 91:10337-10354.

Smith, G.M., Gee, J., and Klootwijk, C.T., 1991. Magnetic petrology of basalts from Ninetyeast Ridge. In Weissel, J., Peirce, J., Taylor, E., Alt, J., et al., Proc. ODP, Sci. Results, 121: College Station, TX (Ocean Drilling Program), 525-545.

Stacey, F.D., and Banerjee, S.K., 1974. The Physical Principles of Rock Magnetism. Dev. Solid Earth Geophys., 5: New York (Elsevier).

Stokking, L.B., Heise, E.A., Pariso, J.E., and Allerton, S.A., 1995. Data report: magnetic mineralogy, major- and trace-element geochemistry, and rock magnetic properties of Hole 504B upper crustal rocks. In Erzinger, J., Becker, K., Dick, H.J.B., and Stokking, L.B. (Eds.), Proc. ODP, Sci. Results, 137/140: College Station, TX (Ocean Drilling Program), 327-338.

Stoner, E.C., and Wohlfarth, E.P., 1948. A mechanism of magnetic hysteresis in heterogeneous alloys. Philos. Trans. R. Soc. London A, 240:599.

Thompson, R., and Oldfield, F., 1986. Environmental Magnetism: London (Allen and Unwin).

Waychunas, G.A., 1991. Crystal chemistry of oxides and oxyhydroxides. In Lindsley, D.H. (Ed.), Oxide Minerals: Petrologic and Magnetic Significance. Rev. Mineral., 25:11-68.

Xu, S., and Dunlop, D.J., 1993. Theory of alternating field demagnetization of multidomain grains implications for the origin of pseudo-single domain remanence. J. Geophys. Res., 98:4183-4190.

Xu, S., and Merrill, R.T., 1990. Toward a better understanding of magnetic screening in multidomain grains. J. Geomagn. Geoelectr., 42:637-652.

Date of initial receipt: 15 August 1994

Date of acceptance: 28 February 1995

Ms 148SR-130 
A

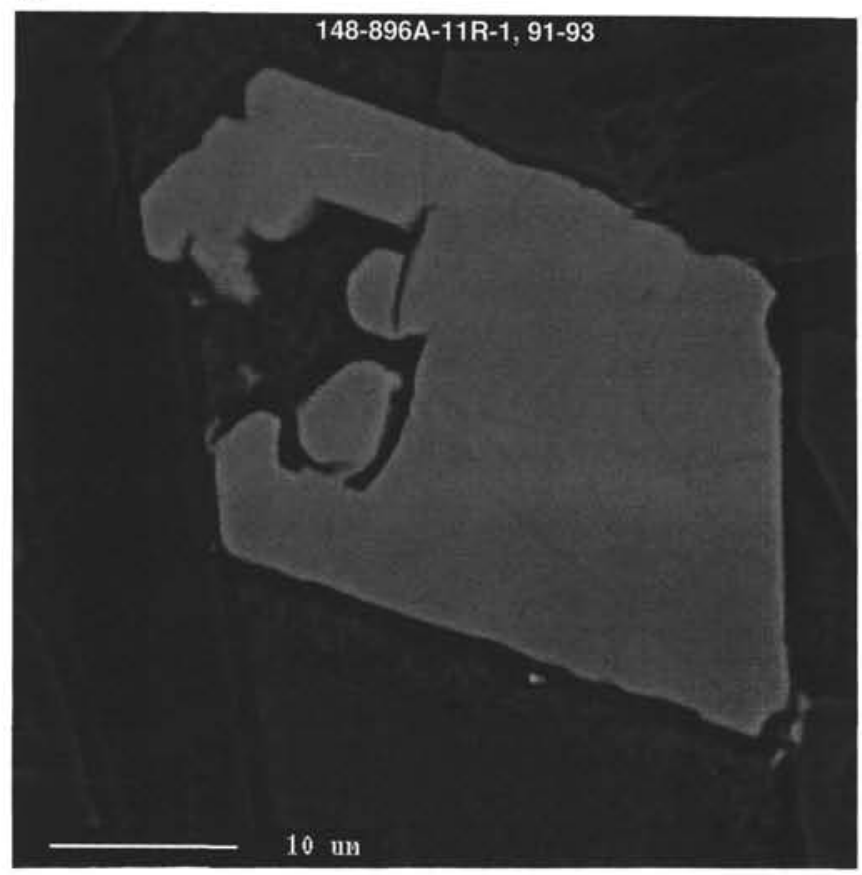

C

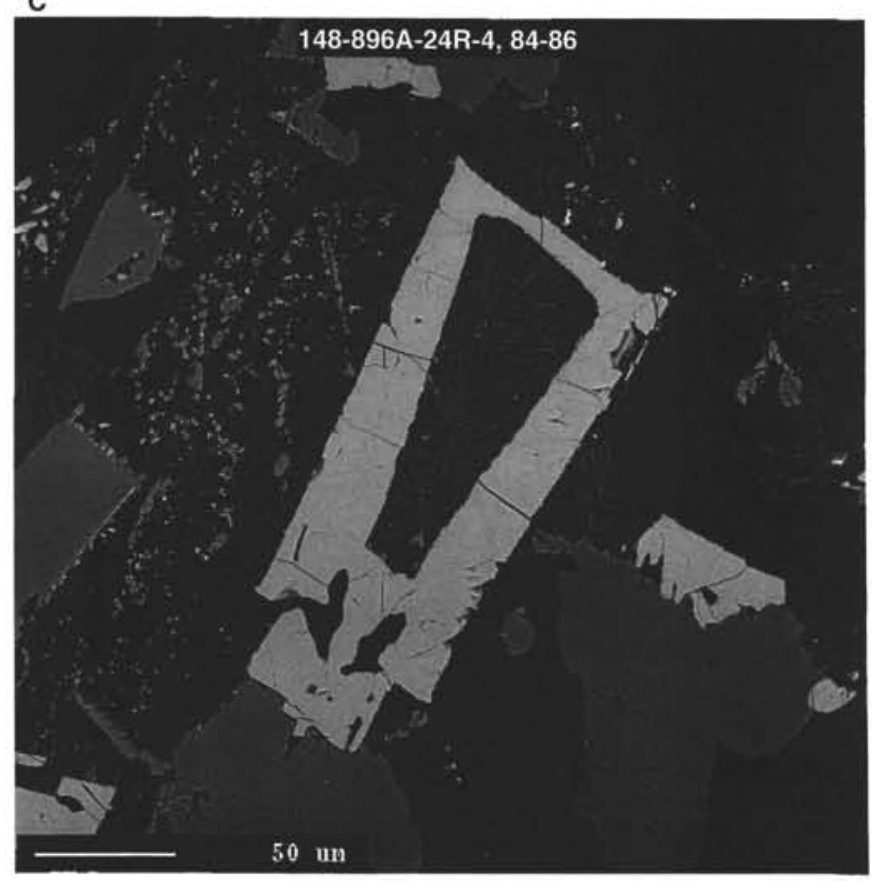

B

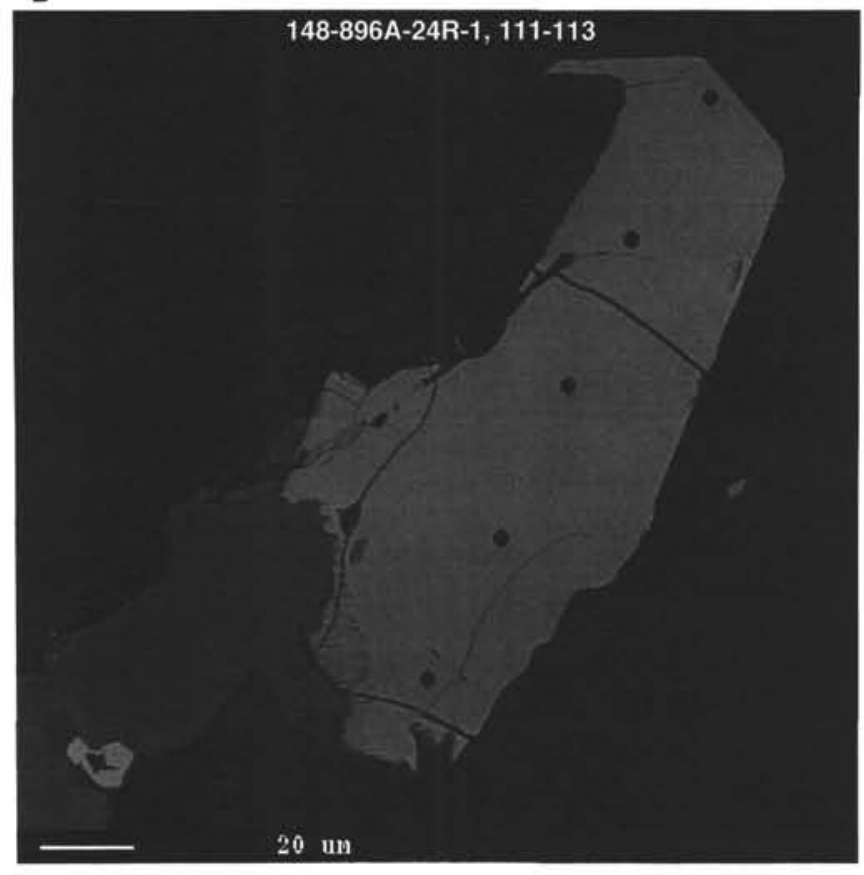

D

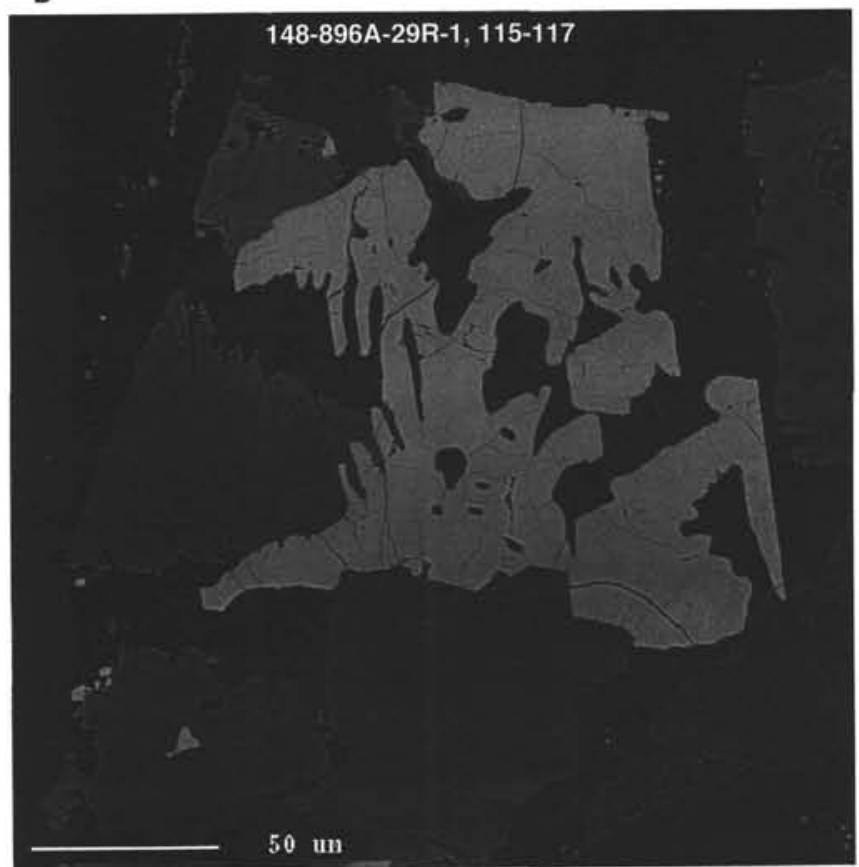

Figure 6. Backscattered electron images. A. Sample 148-896A-11R-1, 91-93 cm. The light-colored grain in the center of the image is titanomaghemite. Dark curved lines cutting the titanomaghemite grain are shrinkage cracks. B. Sample $148-896 \mathrm{~A}-24 \mathrm{R}-1,111-113 \mathrm{~cm}$. The elongate light-colored grain in the center of the image is titanomaghemite. Dark curved lines cutting the titanomaghemite grain are shrinkage cracks. The backscattered electron (BSE) image of this sample was captured after microprobe analyses were performed, whereas the other BSE images presented in Figure 6 were captured before microprobe analyses were conducted. This image, therefore, includes dark spots that were burned in by the microprobe beam. C. Sample $148-896 \mathrm{~A}-24 \mathrm{R}-4,84-86 \mathrm{~cm}$. The large light-colored grains in the center of the image, in the lower left corner, the upper edge, and adjacent to the central grain are titanomaghemite. Dark curved lines cutting the titanomaghemite grain are shrinkage cracks. D. Sample 148-896A-29R-1, 115-117 cm. The large, light-colored, irregularly shaped grain in the center of the image is titanomaghemite. Dark curved lines cutting the titanomaghemite grain are shrinkage cracks. E. Sample 148-896A-30R-1, 134-136 cm. The light-colored, irregularly shaped grain (or grain cluster) in the center of the image is titanomaghemite. Dark curved lines cutting the titanomaghemite grains are shrinkage cracks. 
E
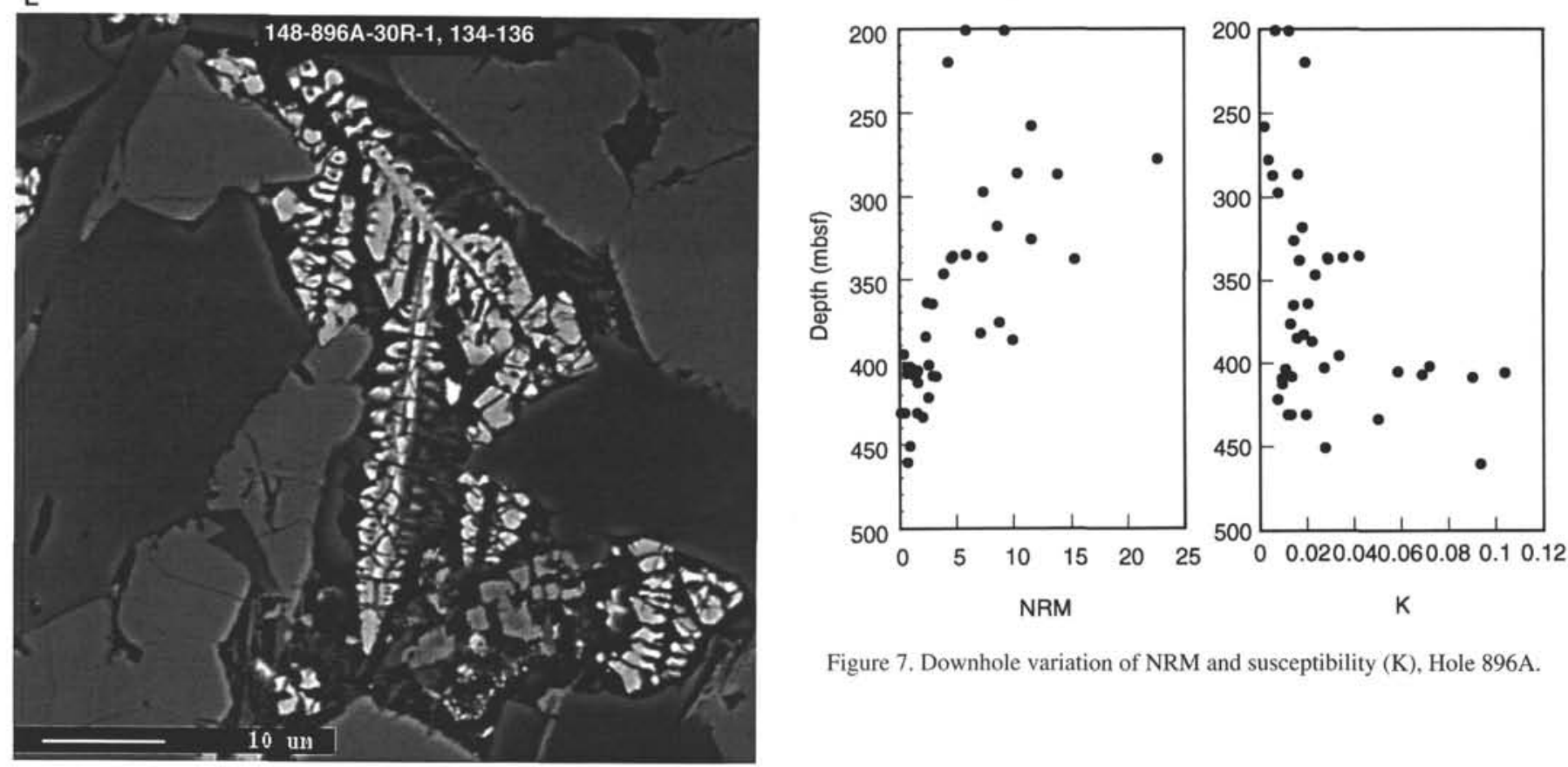

Figure 7. Downhole variation of NRM and susceptibility (K), Hole 896A.

Figure 6 (continued). 